\title{
ASPECTS CLINIQUES ET PATHOLOGIQUES DES ATROPHIES PALLIDALES ET PALLIDO-LUYSIENNES PROGRESSIVES*
}

PAR

\author{
LUDO VAN BOGAERT
}

\author{
(Département de Neurologie et de Neuropathologie de l'Institut Bunge à Berchem-Anvers)
}

\author{
(RECEIVED 10TH OCTOBER, 1946)
}

\section{Introduction}

Nous n'envisagerons pas les troubles extrapyramidaux congénitaux ou apparus dans les premiers mois de la vie, chez des enfants nés avant-terme ou à terme, mais dans des conditions dystociques, et le plus souvent accompagnés d'épilepsie et d'états mentaux déficitaires. Ces cas, del 'étude desquels est née, sans doute, une partie de la pathologie extrapyramidale et qui ont fait l'objet des premières descriptions de l'état marbré (C. Vogt, 1911) et de l'état dysmyélinique (C. et O. Vogt, 1920), sont complexes: aux signes résiduels et peut-être dysplasiques s'y mêlent des états évolutifs qu'il est très difficile, à une période déterminée, de distinguer des premiers, sur le plan clinique et parfois anatomique. Ceux-ci éliminés, l'atrophie pallidale n'est, à l'avant-plan, que dans trois maladies, dont deux sont aujourd'hui acceptées comme autonomes par la majorité des neuropathologistes. La place de la troisième est encore, comme nous le verrons, discutée.

Une atrophie pallidale souvent très importante s'observe dans le cadre des lésions de la chorée chronique. Comme les cas où cette atrophie avait été observée se présentaient cliniquement comme des états de rigidité, on a voulu mettre celle-ci au compte de l'atteinte pallidale (Jakob, 1923), mais Spielmeyer (1926) avait fait remarquer, à juste titre, que les lésions pallidales ne diffèrent, dans de tels cas, que quantitativement des lésions de ce noyau, décrites dans les chorées chroniques, sans rigidité. Cette participation des éléments pallidaux à l'atrophie si caractéristique du strié est d'ailleurs exceptionnelle, même quand l'atrophie striée est très avancée et cela aussi bien dans la chorée chronique familiale que dans la chorée sénile (Alcock, 1936). Ce fait montre, soit dit en passant, que les éléments magnocellulaires de l'un et de l'autre noyau ne constituent pas, comme certains l'ont admis, un système unique. Parmi les "états dysmyéliniques", affections où, à l'étude histologique du strié, on remarque que les fibres myé-

* Lecture given at the National Hospital, Queen Square, on October $9,1946$. liniques aboutissant au pallidum sont particulièrement pauvres, il se trouve des cas familiaux acquis, débutant par des troubles moteurs des membres inférieurs, consistant à la fois en contractures et en mouvements anormaux, évoluant avec des phénomènes athétosiques, choréiques, torsionnistes, se fixant plus tard sous forme d'états de torsion ou de rigidité, avec ou sans troubles mentaux, avec ou sans épilepsie, avec des accès bulbaires ou pseudobulbaires très spéciaux. Le diagnostic clinique de tels cas est souvent très difficile. Hallervorden et Spatz (1922) ont montré, les premiers, que le pallidum et la partie réticulée de la substance noire y sont le siège d'une accumulation anormale de produits pigmentaires du métabolisme, produits qui se trouvent normalement, en petite quantité, à ces niveaux, et dont la nature demeure encore inconnue. Leur précipitation, à ce niveau, modifie l'aspect myélinique, amorce une réaction gliale et peut-être accompagnée, mais pas nécessairement, d'une raréfaction des cellules ganglionnaires. Dans certains cas, d'autres centres extrapyramidaux présentent aussi des altérations minimes, mais c'est l'état pigmentaire pallido-réticulé qui demeure à l'avant-plan. Il n'y a pas, ici, à proprement parler une atteinte sȳstématisée d'un système de neurones: il y a accumulation d'une quantité anormale de produits du métabolisme local. Cet état pigmentaire peut s'observer d'ailleurs au cours de maladies aussi différentes que la neurofibromatose (Gamper, 1929), la démence précoce (Fünfgeld, 1929), les myopathies (Hallervorden, 1928), et l'encéphalite léthargique (van Bogaert, 1940). On peut le voir intriqué à l'évolution d'une atrophie cérébelleuse familiale (van Bogaert, 1946) chez des malades atteints de maladie de Recklinghausen et, dans de telles conditions, sa situation vis à vis de l'affection dégénérative qui l'accompagne est très difficile à fixer. Les limites d'une telle affection sont donc délicates à tracer, puisqu'à côté de cas où les lésions cellulaires sont minimes et où on note simplement une augmentation quantitative des pigments de désintégration on trouve des cas avec des altérations cellulaires pallidales dentelées, cérébelleuses et luysiennes (Vincent et van Bogaert, 1936). Cette 
variabilité des altérations parenchymateuses, accompagnant le dépôt, explique les différences cliniques d'un cas à l'autre, et les discussions dont certaines observations sont, et seront dans l'avenir, l'objet pour ceux qui voudront en établir. le bilan (Eicke, 1940). L'affection individualisée par Hunt (1917) comme "atrophie pallidale progressive", décrite comme une affection du système des grandes cellules putaminales et pallidales, se présente sous forme d'une paralysie agitante juvénile évoluant vers la rigidité. Elle n'est certainement pas une affection du système, mais les faits cliniques n'en demeurent pas moins valables et des cas existent, de cet ordre, qui n'ont rien, à la vérification, d'une forme postencéphalitique (van Bogaert, 1930). Tout comme l'atrophie pallidale des chorées chroniques, comme le type décrit par Lhermitte et MacAlpine (1926), l'affection isolée par Hunt représente une maladie dégénérative complexe où l'atteinte du pallidum n'est qu'un fragment dans une constellation pathologique et analogue à celles dont nous apportons, nous-mêmes, plus loin trois exemples nouveaux. Ces associations, qui sont loin de simplifier nos inductions sémiologiques et de localisation, présentent un intérêt au point de vue de l'histopathologie générale, à peine amorcée, malgré de longues et difficiles recherches, de ces centres.

\section{Méthodes}

Nous touchons ici à une question très épineuse. La littérature des affections extrapyramidales est encombrée d'observations pathologiques- à n'accepter qu'avec réserve. Cela provient tout d'abord de ce que les structures myéliniques, cellulaires, gliales, de ce que le métabolisme lipopigmentaire des différents noyaux présentent des variations physiologiques qui viennent à peine d'être précisées, pour certains âges. On ne saurait, à ce point de vue, assez apprécier l'œuvre de longue haleine entreprise depuis trente ans par les Vogt et leurs collaborateurs,' sur l'anatomie normale des différentes formations optostriées et mésencéphaliques et qu'ils viennent, dans ces dernières années, d'étendre à la pathologie de ces régions (C. et $\mathbf{O}$. Vogt, 1937a et b; Hassler, 1937a et b; Brockhaus, 1938, 1942; C. et O. Vogt, 1942).

On ne reviendra jamais assez, quand on étudie ces régions, à leur iconographie si précise. Mais celle-ci ne suffit pas. La fréquence des variations normales appelle, pour quiconque qui ne dispose pas des mêmes moyens iconographiques qu'eux, des coupes de contrôle traitées par les mêmes techniques que les cas pathologiques. Toute densité cellulaire, qu'elle soit ganglionnaire ou gliale, ne peut être affirmée qu'après comparaison avec ces témoins et après photographie s'il y a le moindre doute . . . i il est si aisé de prendre pour une gliose réelle ce qui n'est qu'une augmentation apparente de la glie cellulaire due à la rétraction du tissu cérébral au cours de l'inclusion. Les raréfactions ou éclaircissements myéliniques doivent être confirmés par les images cellulaires ou gliales correspondantes, car on sait combien un léger excès de différentiation peut tromper les meilleurs observateurs. Nous réservons toutes celles qui sont muettes dans les coupes cellulaires et gliales. On ne retiendra une surcharge lipopigmentaire que si elle est grossière et coexiste avec de vraies altérations du paren- $\bar{c}$ chyme. D'ailleurs tant que nous ne disposerons pas $\mathbb{D}$ d'une méthode suffisamment fidèle de numération des cellules ganglionnaires et gliales, il vaut mieux, pour ne $ㅇ$ pas rendre plus difficiles les recherches de ceux qui nous $Z$ suivront, ne retenir que les lésions suffisamment importantes pour qu'une erreur d'appréciation visuelle ou photographique puisse être sûrement écartée. Les pathologistes de ce pays ont tellement bien senti ce besoin qu'il n'ont pas manqué de méthodes ingénieuses (je pense au travail d'Alcock (1936) sur la chorée sénile, par of exemple) pour numérer les régions des cas témoins et morbides.

L'exploration des cas doit enfin être poussée à tous les niveaux: on est frappé de voir combien d'auteurs, et même des plus en vue, en présence d'un cas avec une sémiologie déterminée se limitent à scruter minutieuse- $\overrightarrow{\vec{N}}$ ment le niveau où ils présument que doit se trouver, $\vec{\nabla}$ dans leurs hypothèses, l'altération et combien ils traitent superficiellement les régions, jugées d'avance, peu intéressantes.

La pathologie extrapyramidale est enrichie d'observations tendancieuses: si les lésions découvertes ne cadrent pas avec nos vues actuelles, une raison de plus pour les retenir, ce sont peut-être celles qui un jour indiqueront la voie de l'interprétation vraie. Sans doute une méthode, comme celle dévèloppée par les Vogt, est-elle longue, demande-t-elle un effort et est-elle techniquement couteuse? Comme toute donnée pathologiquȩ̣ elle ne fixe qu'une étape et malheureusemen $\vec{N}$ l'étape terminale, mais précisément la modesti et la réserve, à laquelle elles nous obligent, n'ontelles pas la valeur d'un enseignement? Dans matériel clinique et anatomique qui, pendant ceई 웅 vingt dernières années, nous a été confié et montrę par des collaborateurs et amis, auxquels nous tenons. à dire ici, une fois de plus, notre gratitude, noưs avons choisi neuf cas, dont huit suivis personnellement par nous pendant leur vie. Ils nous paraissent éclairer d'une certaine manière le problème des atrophies pallidales, malgré les réserves que nous demandent les méthodes actuelles d'investigation pathologique.

La méthode que nous avons utilisée n'est pas, comme celle des Vogt, une étude sur des séries complètes à la paraffine, où une coupe est alternativement traitée par une méthode myélinique, cellulaire et gliale. Nous ne disposons pas du personnel ni du matériel (surtout pendant les années de guerre) permettant un travail aussi parfait au point de vue hodographique et systématique. D'ailleurs, pour des buts histopathologiques, cette technique ne nous suffit pas: elle ne permet pas d'appliquer, au même niveau, les méthodes pour les graisses et les méthodes spéciales pour le neuroglie, du moins actuellement. Nous avons préféré étudier chaque cas, en séries discontinues, à congélation et en incluant quelques grands blocs à la celloïdine pour les contrôles de cyto-architectonie: ainsi seulement, chaque niveau altéré peut-être exploré au point de vue de ses cellules, de ses gaines myéliniques, de ses fibrilles, de sa glie et de ses produits de désintégration. Nous verrons qu'il n'est pas trop de toutes ces méthodes combinées pour ne pas passer à côté d'altérations qui ne sont pas assez décisives avec l'une d'elles seule, et qui ne prennent toute leur valeur qu'à la lumière des autres. De nombreuses photographies d'ensemble sont précieuses car elles seules permettent, par comparaison avec les témoins, de se faire une idée de l'extension des dégénérescences dans l'étendue du noyau: nous les devons au dévouement et à l'art du Dr. A. Dewulf (de Corbeeck-Loo). 
Il n'est pas superflu peut-être de rappeler ici quelques notions anatomiques et histologiques récentes qui permettront de mieux situer les pré-. cisions pathologiques que nous tâcherons de formuler, à propos de chaque cas. Dans l'ensemble des noyaux gris centraux, ce n'est pas arbitrairement qu'on peut considérer le noyau pallidal comme formant une unité systématique.

Il y a une composition cellulaire bien particulière: un seul type de neurones, peu nombreux, plus de cellules d'oligodendroglie (C. et O. Vogt, 1942) que le putamen et réparties plus uniformément. Alors que les grandes cellules striées ont, avec les techniques à l'argent, une structure banale et qu'elles n'ont, comme les cellules corticales, à leur surface aucun des appareils de contact du type des boutons terminaux, les éléments pallidaux sont étoilés, à longs dendrites cernés de nombreux boutons terminaux, et revêtus d'un manteau protoplasmique gliogène épais (Bielschowsky, 1919). Les petites cellules striées ont des dendrites nombreux à axones courts et représentant des cellules d'association; les grands éléments, des dendrites et axones plus puissants qui sont à l'origine probablement des fibres striopallidales: le strié a, par conséquent, la structure d'un appareil complexe de transmission, de régulation (C. et O. Vogt, 1942) et non d'un noyau autonome. Le pallidum a un réseau vasculaire moins riche. (Spielmeyer, 1920). Les cellules pallidales ont peu de fibrilles et elles sont grossières. Il est enfin le siège de dépôts pigmentaires, gras, sidérophiles et pseudocalcaires plus abondants, indicateurs d'un métabolisme particulier (Spatz, 1921, 1922).

Que ces caractères structurels aient une contrepartie fonctionnelle c'est vraisemblable, mais nos connaissances sur la physiologie de ce noyau sont, chez l'homme, à peine amorcées. La thèse de Hunt (1933) homologuant les grandes cellules striées, les grandes cellules pallidales et celles de la substance innommée n'a, en tout cas, pas de base anatomique (Davison, Goodhart et Shlionsky, 1932; C. et O. Vogt, 1942). L'étude des connexions de ce noyau avec les centres supra- et infra-thalamiques est un peu plus avancée qu'à l'époque des Vogt et de Foix et Nicolesco (1925). On doit beaucoup aux recherches récentes de Ranson et Berry (1941), des Mettler (1942), de Papez (1942) et de Glees (1945). Nous n'avons conservé, dans nos descriptions, les abréviations proposées par les Vogt (1942) que pour faciliter les comparaisons de nos microphotographies avec celles de leurs atlas.

Le putamen (Put.) et noyau caudé (N.C.) envoient des fibres aux différents segments du pallidum, fibres qui y pénètrent en rayon de roue: ce sont les fibres striopallidales (f.s.p.). Celles de la partie antérieure du N.C. et de la partie adjacente du Put. se mélangent à celles de la C.I. et atteignent ainsi la partie orale du Pal., pour se jeter dans son segment interne (g.i.) et, pour une petite partie, dans les éléments du noyau entopédonculaire. Les fibres issues des autres parties du Put. vont en g.e. (Mettler). Les fibres de la queue du N.C. pénètrent, sur toute l'étendue de celle-ci, dans le pallidum, les plus antérieures se joignent à celles de la tête du N.C., les postérieures et ventrales passent à travers le $\mathrm{Pal}$. et le pied (P.) pour entrer dans la partie latérale de la S.N. (Mettler). Des fibres relient entr'elles les deux segments pallidaux. Les connexions efférentes du Pal. sont complexes et discutées. Papez (1942) y distingue l'anse lenticulaire (a.l.) (Fig. 1A), le faisceau lenticulaire (f.l.) (Fig. 1c); le faisceau pallido-luysien (f.p.l.) (Fig. 1B), le faisceau thalamique (f.t. ou H.1), le faisceau pallidohypothalamique (f.p.h.) (Fig. 1A) qui se terminent pour leur grande majorité dans le champ H., le C.L., le noyau ventral lateral (v.l. de Papez, v.t.l. des Vogt, a.v. de Ranson), de la couche optique et dans l'hypothalamus. Elles ont pour origine des cellules pallidales. La composante pallidale de l'a.l. a pour origine le g.i. surtout ventral (Fig. 1A). Après un court trajet en dedans, ces fibres se replient en arrière autour du bord interne de la C.I., et rejoignent celles du f.l.; le f.l. a, aussi, pour origine principale les cellules du g.i. Ses fibres s'accumulent à la face externe de la partie moyenne de C.I. constituant la "zone limitante" pénètrent à travers de la capsule dans le sens dorso-médian et forment à la face interne de la C.I., le f.l., puis se dirigent en dedans pour rejoindre l'a.l. Les deux faisceaux se dirigent alors à nouveau en arrière et passent dans le champ prérubral (H.). Cette partie descendante de leur trajet est souvent appelée H.2. Au moment de passer dans le champ H., au contact de la face latérale du faisceau mamillo-thalamique, les fibres se retournent en grande partie en haut et en dehors constituant alors le faisceau thalamique ou champ H.1. Ce faisceau thalamique pénètre immédiatement dans le bord interne de la partie antérieure de v.l. et s'y distribue en dehors et en avant. Au moment de se retourner pour former H.1, quelques fibres fines sont émises en dehors vers le champs $\mathrm{H}$., où se trouvent précisément les noyaux du champ de Forel et la zone incerta (Z.I.). Les fibres du N.c.F. (ou f. prérubral) directes et peut-être croisées forment la partie frontale du secteur médio-dorsal de la capsule du N.R. (Fig. 1C). Des fibres qui- constituent l'a.l., un faisceau isolé plonge verticalement vers le noyau hypothalamique ventro-médian (Ranson, 1943). Des fibres partent de g.e. et n'empruntent pas le champ H., passent à travers la capsule du C.L., pénètrent dans le C.L. et constituent la partie ventro-latérale de la capsule (Ranson, 1943); d'autres viennent de g.i. à travers la capsule (Papez, 1942) et entrent dans la composition de la partie médio-latérale de la capsule (Fig. 1B). La partie dorsale de sa capsule du C.L. est constituée par les fibres du f.l. qui l'abordent (Fig. 1C).

Les efférences allant au N.R., à la S.N., au noyau de Darkschewitsch (N.Da.), au noyau interstitiel (N.i.) et à la commissure postérieure (c.p.) soit par H.1 ou H.2, postulées encore par les Vogt (1920) et les Mettler (1942), sont réservées par Ranson (1943). Les recherches expérimentales les plus récentes de Glees (1945) chez $M$. rhésus semblent confirmer l'ensemble des connexions que nous venons d'esquisser. Le contingent afférent est représenté par les fibres strio-pallidales (f.s.p.) (Fig. 1B), mais surtout par le système thalamo-pallidal (f.th.p.) issu des mêmes noyaux qui projette sur le strié (Mettler, 1942). Ce système appelé encore pédoncule thalamique inférieur (i.p.) (Papez, 1942) s'étale le long du bord médian de la C.I., la traverse, pénétre dans le Pal. par son bord dorsal et antérieur. Il a pour origine la partie médiane des noyaux médian de la C.O. (m.e. de Papez), sous-médian (ou médian ventral) et peut-être du noyau antérieur. Il se divise en deux courants: un antérieur qui s'incurve en avant et en dehors, pénètre dans les lames pallidales (l.p.) et s'épanouit dans le pallidum, un courant postérieur qui reste dans l'hypothalamus, s'étendant en arrière dans le Z.I., entre le f.l. et H.1. Au cours de ce trajet ses fibres pénètrent dans le pallidum postérieur.

De la physiologie de ce noyau nous ne savons rien de certain et on peut lui appliquer ce que les Ranson (1943) écrivaient encore récemment à propos du corps strié en général: "Le corps strié est généralement considéré comme un chainon très important de la voie motrice extrapyramidale, mais il est certain que beaucoup de ce qui a été écrit sur cette partie de la voie qui descend du corps strié aux centres inférieurs du cerveau est erroné. La fonction du corps strié et le trajet des voies descendantes par lesquelles il influence l'activité spinale sont toujours, en grande partie, inconnus." 
L'étude des connexions anatomiques respectives du pallidum et du strié suggère à Papez (1942) que les voies motrices d'origine pallidale se continuent par le noyau rouge, constituant un système d'innervation musculaire, engagé spécialement dans les phases de contraction de l'activité musculaire, alors que le système strié, dont les voies passent par le S.N., le tegmentum, l'olive inférieure (et qui se trouve, par ce dernier échelon, en contact avec le cervelet), serait un appareil de contrôle et de maintien du relâchement musculaire. Le circuit cérébelleux est embrayé dans les deux systèmes. Nous en parlerons encore à la fin de ce travail.

\section{L'atrophie pallidale pure}

Nos deux premières observations concernent deux cas d'atrophie pallidale pure et progressive survenant chez deux frères. Le premier cas avait été étudié avec le Dr. L. De Vos en 1933 et publié, à cette époque, sous ce titre. Nous le rappellerons brièvement car il fut publié dans une revue pas toujours accessible.

La famille $H$. compte six enfants. Le père est actuellement atteint de tuberculose pulmonaire et a subi deux pneumothorax. La mère est agée de 46 ans et a été opérée récemment d'un goître. Les grands parents paternels ne présentent aucune affection nerveuse décelable et sont apparemment normaux au point de vue psychique. Ils ne sont pas consanguins. Du mariage de Marcel $\dot{H}$. et Eudoxie L. sont issus Marcel, mort à six mois d'une infection grippale; David, qui fit l'objet de notre observation $I$ et qui est décédé à l'âge de 10 ans en 1933; Marie, agée de 21 ans, bien portante; Angeline, agée de 18 ans, bien portante, présentant cependant une forte cyphoscoliose et des pieds creux bilatéraux avec aréflexie tendineuse aux deux membres inférieurs; Anna, agée de 14 ans, bien portante; et Louis, né en 1934 et qui fait l'objet de notre observation II.

Chez David (Observation I) s'est développé, à l'âge de 5 ans, un syndrome d'hypertonie progressive de la jambe puis du bras droit en même temps qu'il présentait un retard marqué d'évolution intellectuelle. L'hypertonie devint bilatérale, en l'espace des quatre premières années, mais demeura moins intense à gauche. La parole devint lente et monotone; il présenta des troubles de la déglutition puis apparurent de grands mouvements d'aspect athétosique mais avec une composante torsionniste et rythmique et des déplacements de grande amplitude autour de l'articulation scapulo-humérale et parfois coxo-fémorale. La brusquerie et l'ampleur de ces mouvements variaient avec les jours. Ils étaient beaucoup moins typiques et moins amples à gauche. Pendant les dernières semaines de la vie les mouvements deviennent de plus en plus amples et violents, il y eut du rire et pleurer spasmodique. Il existait encore un spasme permanent d'ouverture de la bouche. Il n'était presque plus possible de le nourrir; finalement, la tête elle-même participa aux mouvements choréo-athétosiques.

Dans l'interprétation de ce cas nous avions été très prudents; les parents invoquaient, au début 7 de l'affection, une infection mal déterminée et

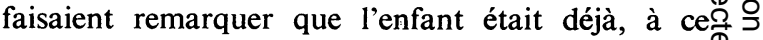
moment, un arriéré. La présence de quelques尺 réactions gliales marginales dans la région ventricu laire et chiasmatique pouvaient être retenues dans $\bigcirc$ ce sens. Cependant sur le caractère systématisé efO ancien de l'atrophie pallidale, il n'y avait pas de

Famille $\mathbf{H}$.

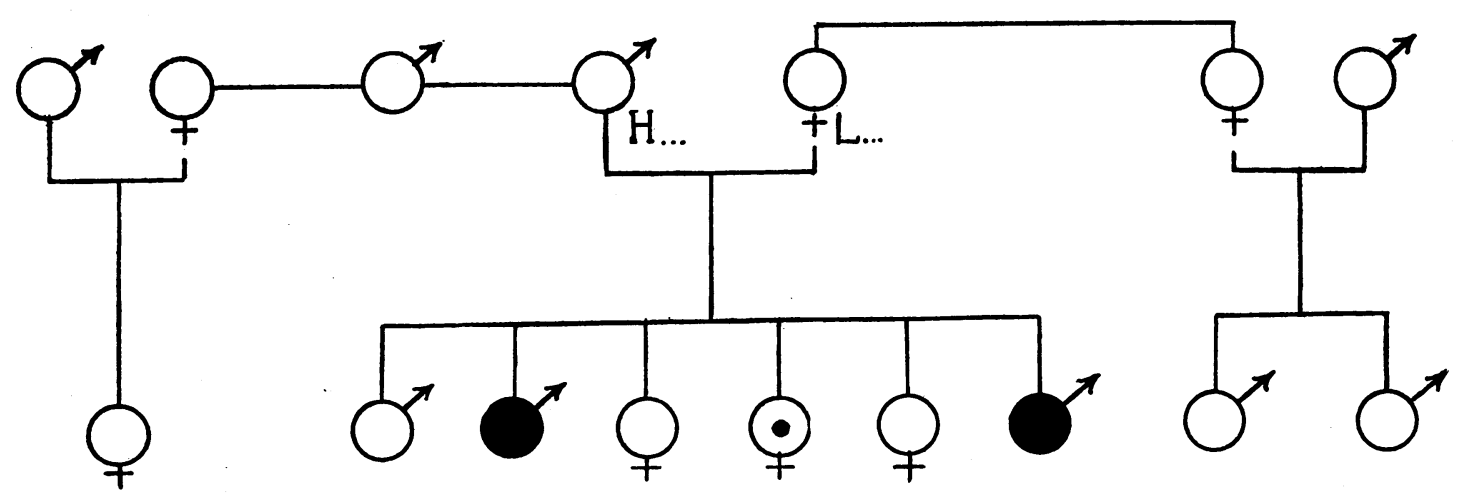

Choréo-athétose progressive à évolution rapide.

Fragment hérédo-dégéneratif de la série Friedreich. 


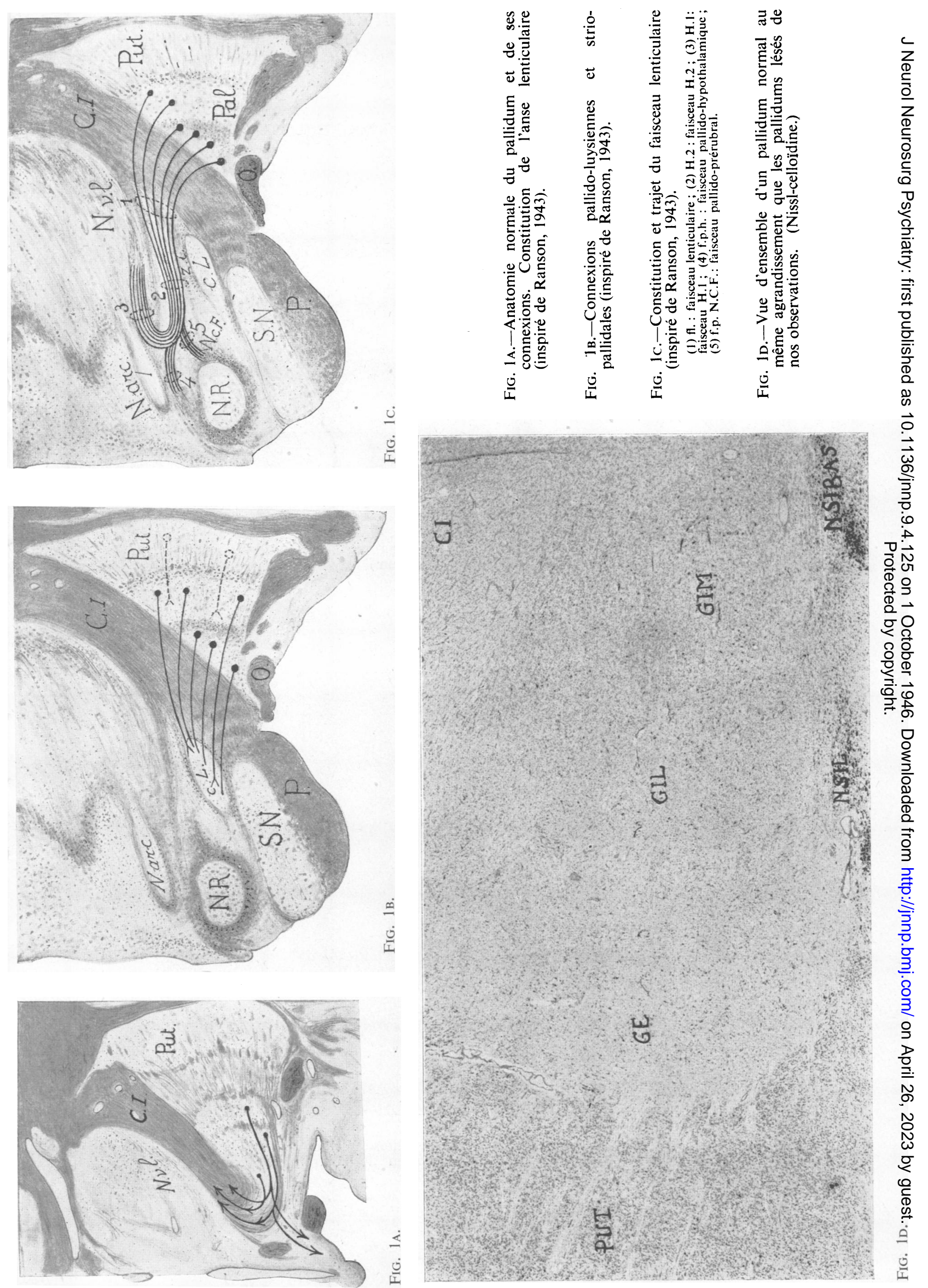



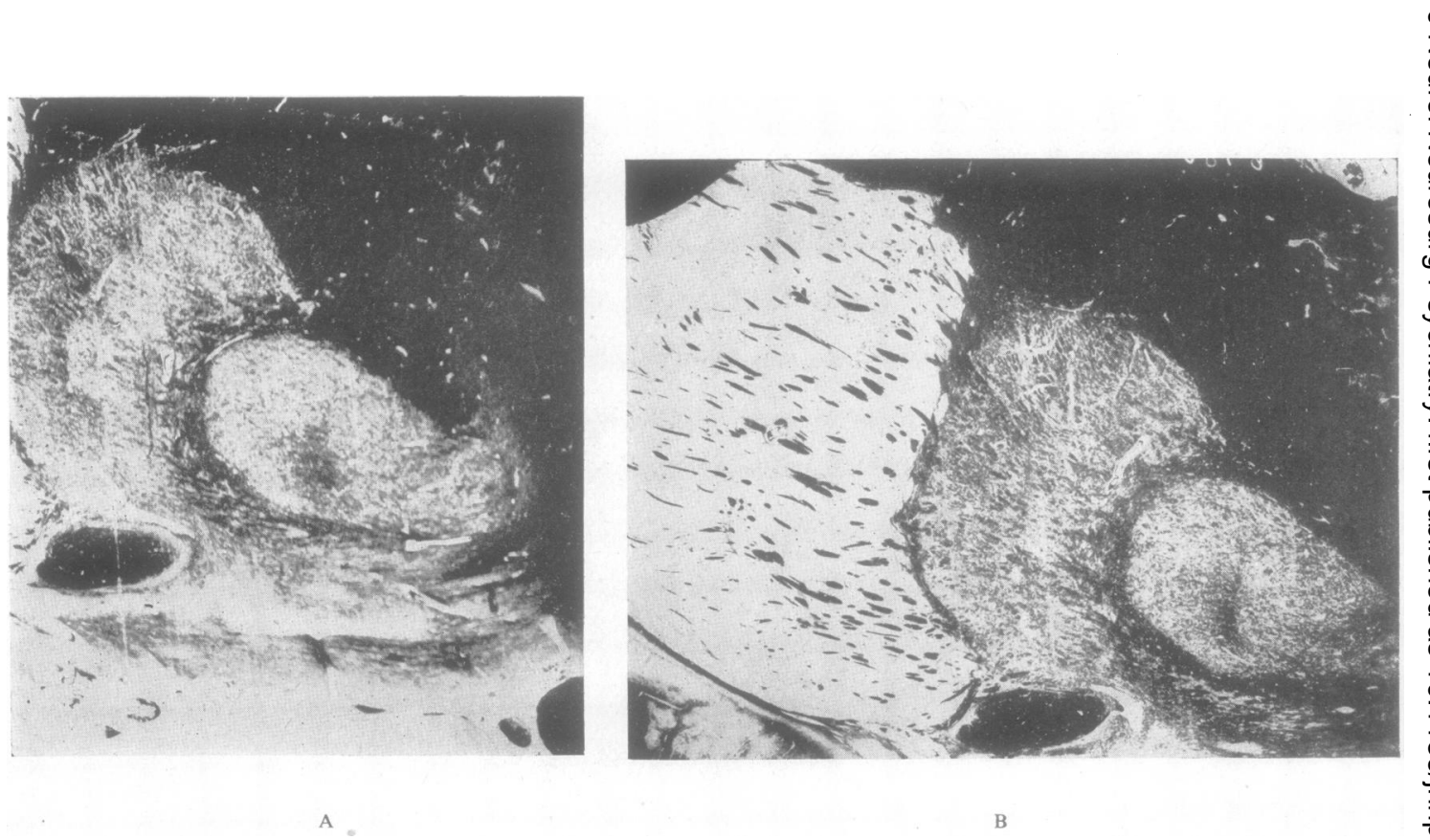

FIG. 2A.-David H. (Obs. I). Légère pâleur de g.i. et g.e. (Spielmeyer-congélation.) B.-Louis H. (Obs. II). Légère pâleur de g.i., meilleure conservation de g.e. (Spielmeyer-congélation.)

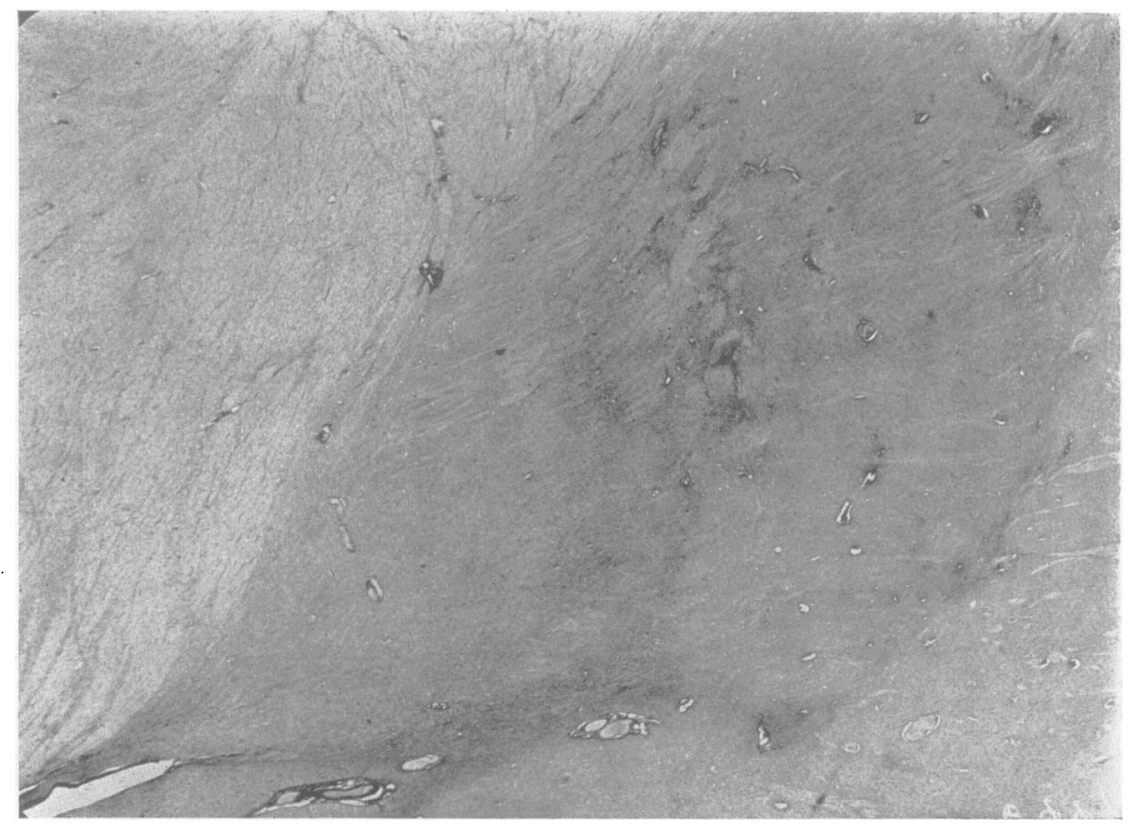

FIG. 3.-Louis H. (Obs. II). Gliose fibrillaire diffuse surtout nette en g.e., g.i.l., moins dense en g.i.m. On voit l'imprégnation de l'a.l. (Holzer-congélation.) 

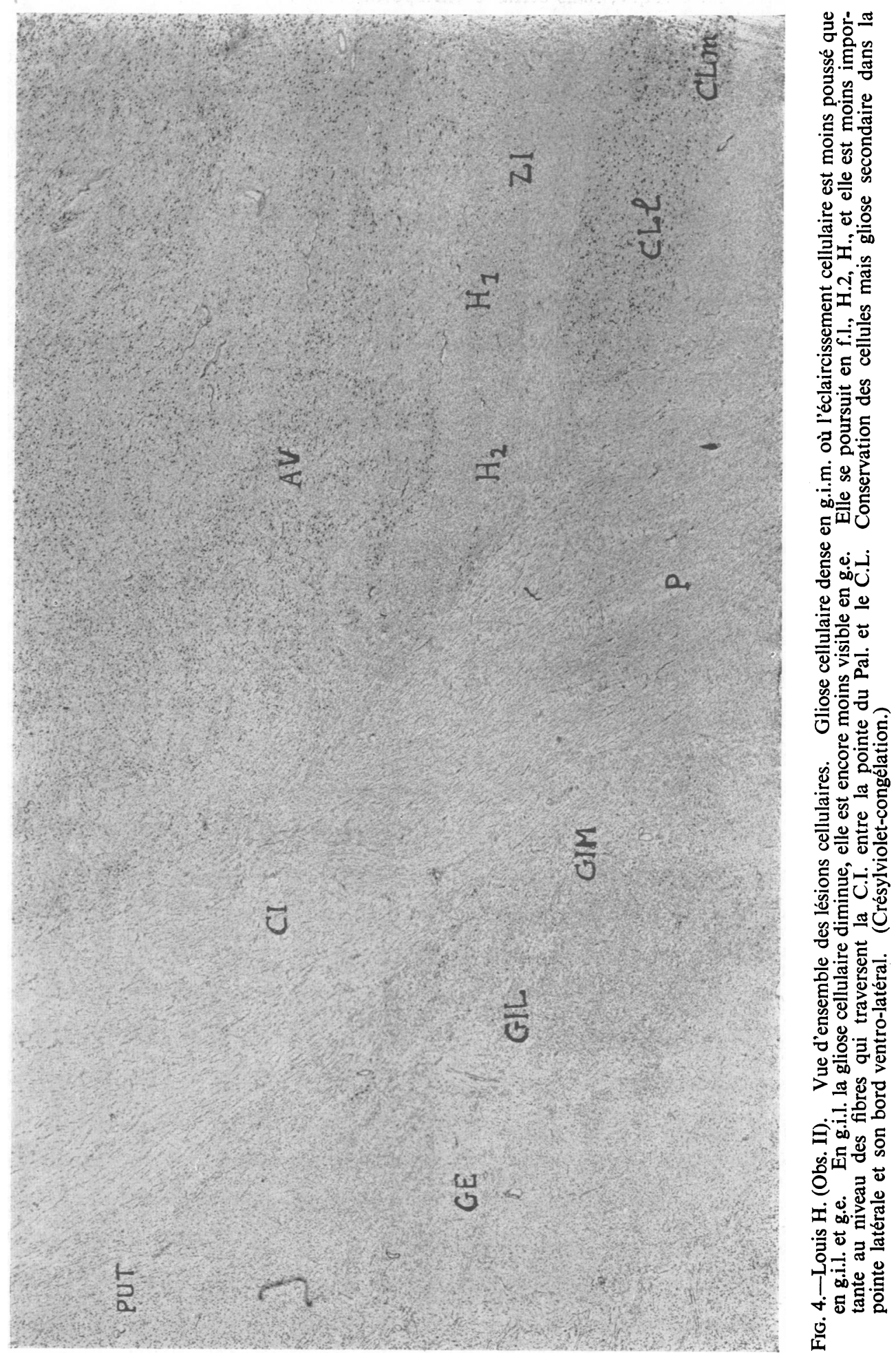
doute. Nous étions tentés de la considérer comme une lésion indirecte, peut-être en relation avec l'ancien incident infectieux invoqué, mais certainement dégénérative.

Son frère Louis (Observation II) était encore bien portant, en 1938, quand nous avions revu la famille, intrigués par la cyphoscoliose, l'aréflexie tendineuse et les pieds creux de la fille Angeline. L'affection débuta, chez Louis, en 1939, à l'âge de 6 ans, par un "tic" de la jambe droite: le pied droit était posé en varus'équin et la marche s'accompagnait souvent d'un mouvement bizarre de circonduction du pied autour de la pointe appuyée sur le sol; puis, peu à peu, apparurent des spasmes dans le bras droit avec une rotation de l'épaule en dedans et à gauche et une rotation de la nuque à gauche.

D'une observation prise à ce moment (1939) par le Dr. De Vos, nous reprenons les notes que voici: "Les mouvements sont moins brusques et moins amples que chez David, mais ils ont les mêmes caractères." De l'examen pratiqué le 9 février 1943, nous retiendrons les détails suivants: Quand l'enfant est couché au lit, tout à fait au repos, on observe qu'une contracture en demiflexion-hyperpronation du membre supérieur droit, la main en hyperflexion sur l'avant-bras, les doigts étendus, le membre inférieur droit est parfois à demifléchi, le pied en varus équin. La même attitude s'observe mais moins nette au membre inférieur gauche. Le membre supérieur gauche est intact apparemment. Des que l'enfant se redresse, la contracture se renforce et apparaissent des mouvements choréo-athétosiques de l'extrémité avec des mouvements de moulinet du bras autour de l'articulation de l'épaule. Ces mouvements sont souvent amples. Le pied montre des mouvements athétosiques en hyperextension palmaire avec éventail des orteils. La marche est digitigrade mais elle est, dangereusement, interrompue par les kinésies reptatoires des deux membres supérieurs et, parfois, par une rétraction brusque de la jambe droit sur le bassin. L'enfant se tient, alors, debout sur le pied gauche contracté en varus équin et tombe. Pas de signes pyramidaux; sensitifs, ni des nerfs craniens. Les fonctions cérébelleuses ne peuvent être explorées à cause des décharges motrices. Une ponction lombaire montre 6 cellules, 0,38 d'albumine pour cent et un B.W. négatif.

Pendant l'année 1944 les mouvements sont devenus plus violents, plus brusques et ont atteint quatre membres, grimacéries de la face avec mouvements bruyants de déglutition et de succion. La déglutition est, par moments, très pénible. La marche est devenue impossible et l'état de la nutrition est mauvais. Il succombe le 20 décembre 1944.

L'histoire clinique de ces deux frères est strictement superposable: début par les membres droits, atteinte successive des membres gauches et de face, mélange de contractures et de mouvement choréoathétosiques dont quelques uns à type torsionniste et très amples, absence de troubles pyramidaux et sensitifs. Seuls les deux enfants masculins sont atteints. Les filles sont indemnes; l'une d'elles présente cependant un complexe hérédo-dégénératif, fragment d'une maladie de Friedreich. Si, chez David, nous avions incriminé une infection très bénigne, sur les détails de laquelle toute précision anqume d'ailleurs, et survenue avant l'apparition des premiers spasmes à la jambe droite, il n'en est plus de même chez Louis, où le début a été sournois et progressif.
Les lésions histologiques (Fig. 2A et B) sont dans les deux cas exactement superposables mais plus importantes dans le second.

Autopsie.-L'autopsie viscérale et nerveuse du cas II fut faite dans des conditions très pénibles du bombardement d'alors. La moelle ne put être prélevée qu'au niveau du segment cervico-dorsal. Le cadavre, d'une maigreur extrème, était dans un excellent état de conservation. Dilatation du cœur droit, foyers de bronchopneumonie dans toute l'étendue du poumon droit; péricholécystite; foie et rate de volume et d'aspect normaux; grosse dilatation gastrique avec quelques suffusions hémorragiques sous-péritonéales. Quelques suffusions hémorragiques s'observent aussi au niveau du cœcum et de l'iléon. Kyste de l'épidyme à droite. Le cerveau n'offre aucune anomalie de structure; poids: 1015 grammes. Après durcissement, les coupes verticotransversales ne montrent rien de particulier non plus. Le pallidum parait assez petit. Le putamen et le noyau caudé ont leur taille habituelle. Léger degré d'hydrocéphalie à vacuo.

Etude histopathologique.-Technique: Sur coupes à congélation-Nissl, Holzer, Spielmeyer, rouge écarlate, Bielschowsky-Reumont, Perdrau.

Écorce: Pas de lésions cellulaires systématisées. Une gliose un peu plus abondante qu'habituellement s'observe dans les couchès V-VI, dans la région frontale et motrice au niveau des trois niveaux prélevés. Le centre ovale et le corps calleux ne montrent aucune particularité.

Noyaux gris centraux: Le noyau caudé, le putamen la capsule interne, l'avant-mur, le trigone, la bandelette. optique, la commissure blanche antérieure n'offren $\bar{B}$ aucune lésion. Le pallidum est un peu pâle qu'habitu宋 ellement sur les coupes qui passent par le plein développe气 ment des noyaux gris centraux et de la région du tubero L'a.l. est beaucoup plus pâle; l.p.i., 1.p.e. sont trèş éclaircies, et g.i. est particulièrement appauvri dans ses fibres myéliniques propres; g.e. est moins touché. Le pilier antérieur du trigone est bien imprégné. Le noyab̆ amygdalien n'offre aucune anomalie. Sur une coupe्. plus postérieure, le pallidum apparait plus petit qu'habitu-ellement mais bien impregné. L'a.l. est légèrement éclaircie. Le pied, le locus niger, le corps de Luys ont leur structure normale. La couche optique montre seulement une dépression profonde au niveau de la partie du noyau interne qui borde le ventricule médian. Les coupes au Holzer montrent elles aussi des lésions pallidales importantes: tout le territoire du pallidum est couvert d'une gliose fibrillaire ancienne. On ne voit que peu d'astrocytes de grande taille. La fibrose gliale est fortement renforcée autour des vaisseaux et au niveau des lames médullaires. Elle est particulièrement dense dans le f.l. et l'a.l. où elle est encore en pleine évolution. Elle est plus dense en g.i.l. et g.e., moins. en g.i.m. (Fig. 3). Le Put. est intact. On retrouve à la partie adjacente de la C.I. une gliose assez nette des. fibres de la zone limitante, puis, plus en dedans, la gliose du f.l. Le faisceau mamillo-thalamique est intact. Un grand nombre de fibres traversent le pied et répondent au fibres efférentes du pallidum présentant la même gliose. Le faisceau telencéphalique médian est plus impregné que normalement. Le corps mamillaire et la couche: optique sont intacts. Un aspect analogue se retrouve I dans la coupe plus postérieure: gliose en évolution de 1'a.1., de 1.p.i. et 1.p.a. de la bandelette optique voisins. La gliose prédomine ici sur g.i. Les coupes $N$ cytologiques sont les plus démonstratives. Le corps de Luys est intact au point de vue cellulaire quoiqu'il présente une certaine gliose particulièrement au niveau de sa partie latérale. Le pallidum offre une raréfaction marquée de g.e. et g.i.l:, avec une gliose cellulaire importante, mais cette gliose est la plus dense en g.i.. où les cellules sont beaucoup moins éclaircies (Fig. 4).. On distingue, en travers de la C.I., une bande de gliose cellulaire joignant le pallidum au C.L. et correspondant à la bande semblable observée dans les Holzer. Cette 
bande répond à la dégénérescence des faisceaux efférents directs du pallidum. La gliose y est au moins aussi dense que dans le f.l. Les noyaux thalamiques paraissent indemnes. Une coupe tout à fait postérieure montre une conservation relative des cellules les plus caudales du g.e., avec gliose cellulaire dense, l'intégrité du Put., du N.R. avec gliose secondaire, une légère gliose de la S.N.r., l'intégrité de la S.N.c. et du noyau tegmental latéral (N.tg.l.).

Pédoncule cérébral: Intégrité du noyau rouge, de la substance noire, du pied du pédoncule et des formations périaquéductales pigmentaires. Une légère gliose de la partie réticulée du locus niger peut être retenue. Cervelet: Pas d'altérations de l'écorce ni des noyaux centraux. Pont bulbe et moelle: Rien à retenir. Moelle cervicale: Deux ganglions cervicaux sont examinés: intacts. Des fragments du nerf radial et sciatique poplité exierne, du deltoïde, des muscles jumeaux ont été prélev :s mais ne montrent rien de particulier.

La lésion essentielle est, ici, l'atrophie pallidale. L'image de celle-ci n'est pas uniforme dans les coupes myéliniques et cellulaires. Tout d'abord, n'était-ce l'éclaircissement marqué de l'anse lenticulaire, on pourrait hésiter sur la pâleur des segments externe et moyen. C'est surtout en avant, celle du segment interne qui ne fait pas de doute, or, c'est, dans les coupes par la méthode glio-fibrillaire, à ce niveau que la gliose est la moins dense. C'est là aussi que les cellules sont relativement les moins éclaircies alors que les segments externes, moins démyélinisés, moins riches en cellules gliales, sont les plus raréfiée, proportionnellement, au point de vue cellulaire. Le corps de Luys est indemne: on n'y observe qu'une gliose secondaire à l'atteinte de la connexion pallido-luysienne et surtout marquée dans son segment ventrolatéral. Les autres formations extrapyramidales sont rigoureusement indemnes. L'importance des fibres efférentes directes du pallidum (a.l.) à travers la C.I., en dehors du f.l., est frappante. Notons encore la présence d'une gliose cellulaire dans la région orale du N.R., dans le N.tg.l. et la S.N.r. Ces lésions se superposent, à peu de chose près, aux données de notre premier cas où la raréfaction cellulaire du segment externe contraste avec la discrétion de la gliose. Ici le segment moyen est mieux conservé et moins infiltré de cellules gliales. Dans ce premier cas l'écorce n'était pas absolument intacte au niveau des deuxième et troisième couches.

Chez ces deux frères, à un tableau choréo-athétose progressive avec des spasmes parfois torsionnistes et dont l'ampleur évoque l'hémiballisme, débutant chez tous deux à droite, correspond une dégénérescence systématisée du pallidum atteignant très gravement les segments externes et un peu moins gravement le reste du noyau. Ce processus respecte les formations extrapyramidales voisines et même le corps de Luys, en dépit des connexions étroites de ce noyau avec le pallidum. Le locus niger est intact si l'on réserve la très minime gliose notée en quelques endroits de la zone rouge. Cette

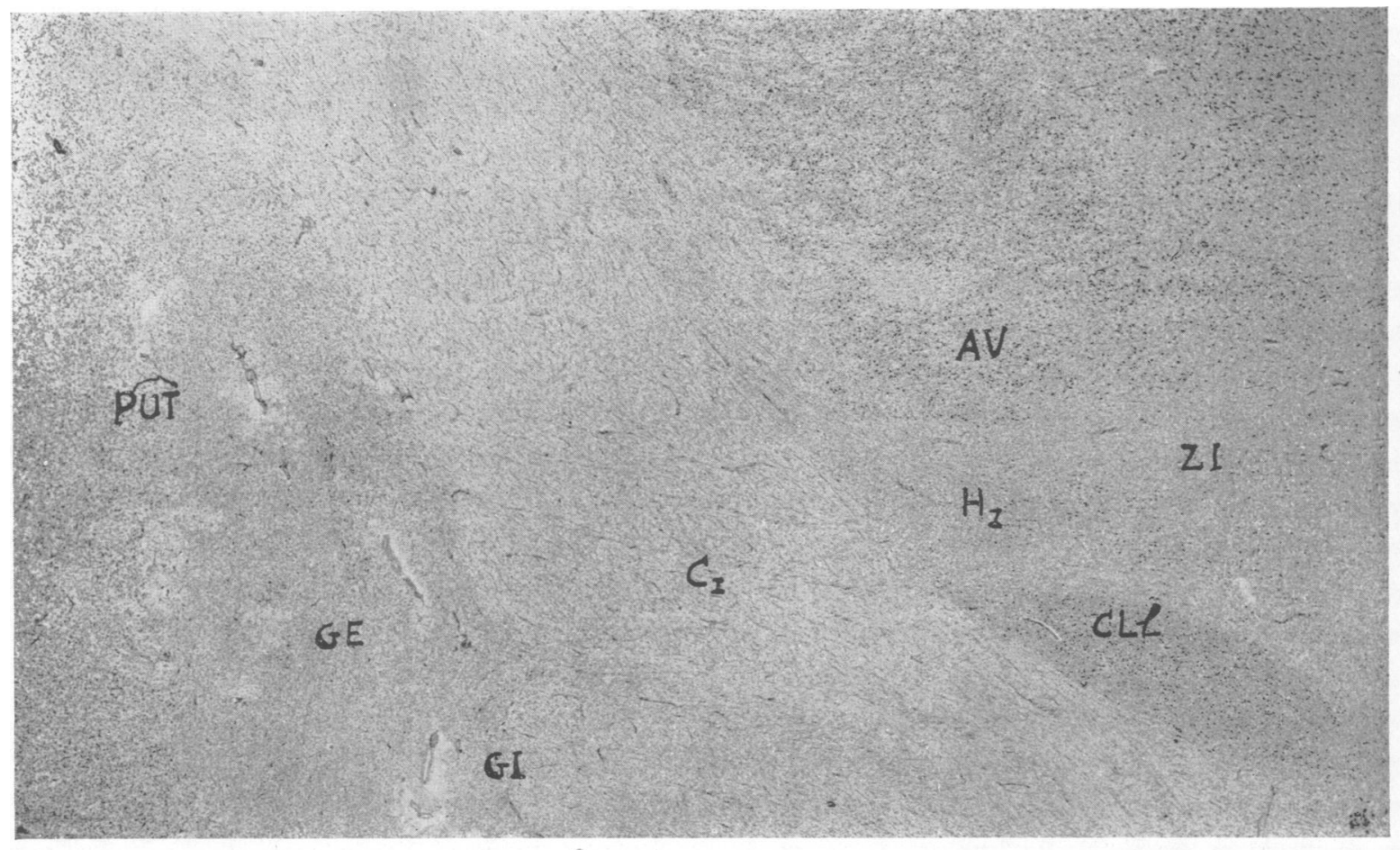

FIG. 5.-Hanc . . (Obs. III). Raréfaction cellulaire égale en g.e. et g.i. Gliose modérée et uniforme. On la poursuit sous forme d'une bande dans la C.L., puis au-dessus de cette bande sous forme d'une plage moins bien limitée répondant au f.l. Le champ H.2, la zone limitante et $H$. présentent une gliose franche. Le C.L. est intact au point de vue cellulaire, mais le siège d'une gliose dense dans toute l'étendue de ses deux tiers externes. (Crésylviolet-celloïdine.) 


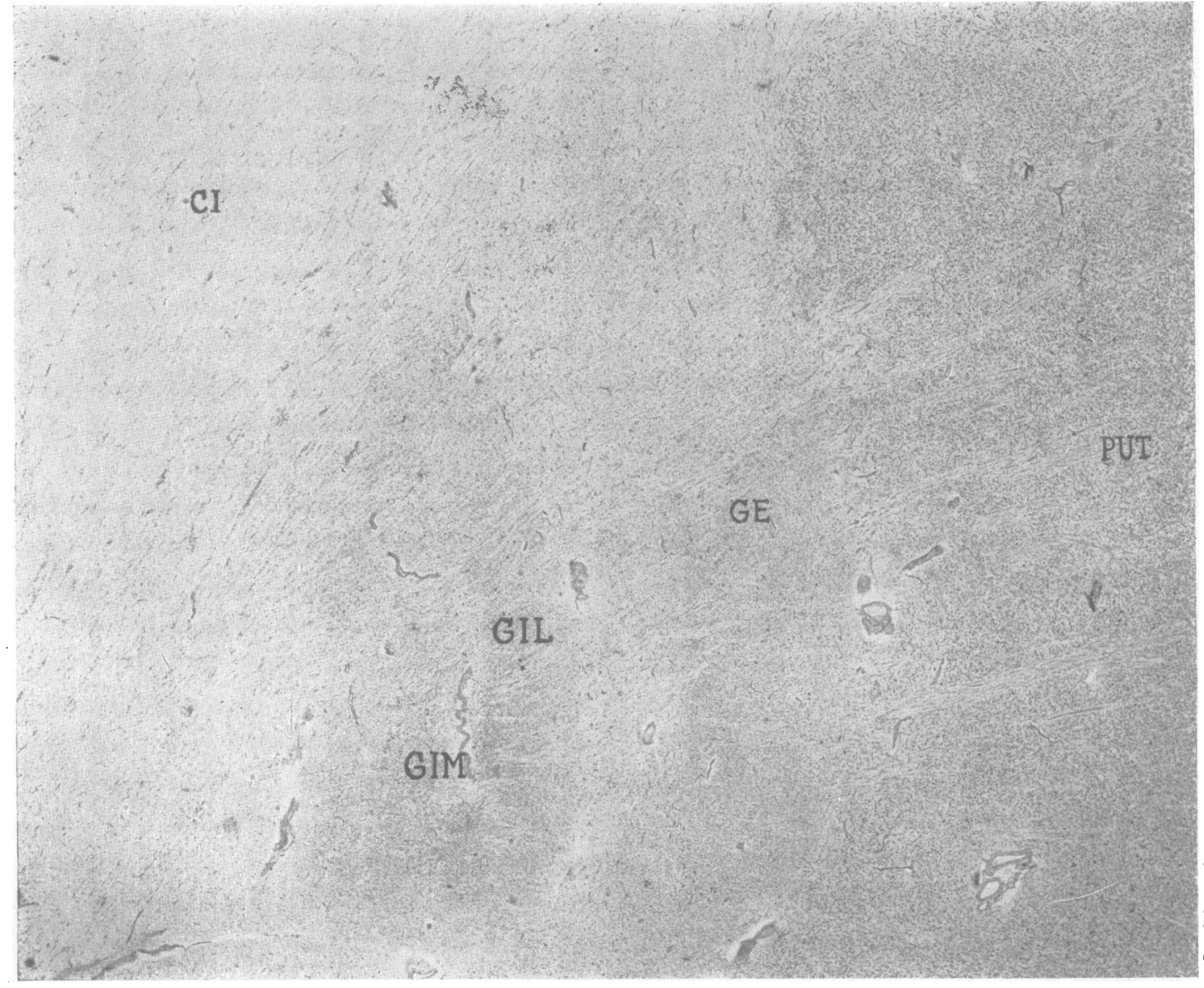

Fig. 6.-Hanc ... (Obs. III). Raréfaction cellulaire plus marquée en g.i.m. que dans g.i.l. et g.e. Gliose cellulaire surtout dense en g.i.l. La gliose parait plus dense dans les parties ventrales du noyau mais peut-être est-ce un artefact, la coupe étant un peu plus épaisse dans sa partie inférieure que supérieure. La gliose cellulaire de l'a.l. est peu visible. (Crésylviolet-celloïdine.)

dégénérescence ne s'accompagne d'aucune altération, ni prolifération vasculaire, mais d'une gliose isomorphe particulièrement distincte au niveau des voies issues du noyau et ne laisse, sur place, aucun produit dégénératif. Chez ces deux frères, le tableau de la choréo-athétose double progressive s'accompagne d'une ébauche de torsion axiale vers la gauche. C'est cette particularité clinique qui nous a rappelé une autre de nos observations.

L'observation Hanc . . . (Observation III) publiée, avec G. Bouché (1935) dans ses détails, concernait un jeune homme de 21 ans chez lequel s'installa, à la suite d'une crise rhumatismale, une contracture en varus équin du pied gauche, s'étendant progressivement au bras et à la jambe. Au bout de cinq ans, apparurent des crises de torsion avec douleurs et finalement un tremblement rythmique et des mouvements choréo-athétosiques. Il mourut d'une septicémie au cours d'une endocardite lente. L'étude histopathologique montra à côté des déterminations inflammatoires d'une méningo-encéphalite à petits foyers, un processus systématisé d'atrophie pallidale bilatérale. Nous avons développé, avec Scherer (1933), les considérations que suscite ce cas au point de vue de la physiopathologie des états de torsion.

Une étude histopathologique plus profonde de ce cas au point de vue de la systématisation intranucléaire du processus dégénératif dans l'hémisphère droit peut avoir son intérêt, si on le compare à nos cas I et II, dont l'étude n'a pas été aussi poussée en raison des circonstances auxquelles nous avons déjà fait allusion.

La coupe la plus caudale passe par la partie postérieure du corps de Luys et du locus niger et montre une raréfaction massive avec gliose modérée dans toute l'étendue de g.i. et g.e. Le C.L. et la Z.I. sont intacts au point de vue cellulaire. Les noyaux du champ $H$. et l'anse lenticulaire sont atrophiés. Sur les noyaux thalamiques eux-mêmes nous ne pouvons nous attarder ici: le noyau arqué nous a semblé moins dense et le siège d'une gliose cellulaire. La coupe suivante passe en arrière des corps mamillaires: la raréfaction est la plus dense ainsi que la gliose dans g.i. Dans celle qui passe par la partie caudale du corps mamillaire, on distingue nettement une raréfaction moins grave dans g.i.l., avec une gliose 


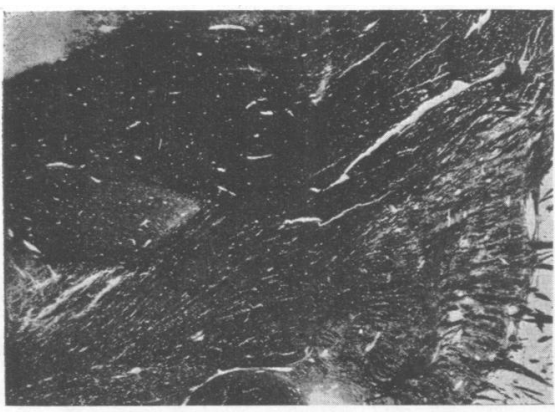

A

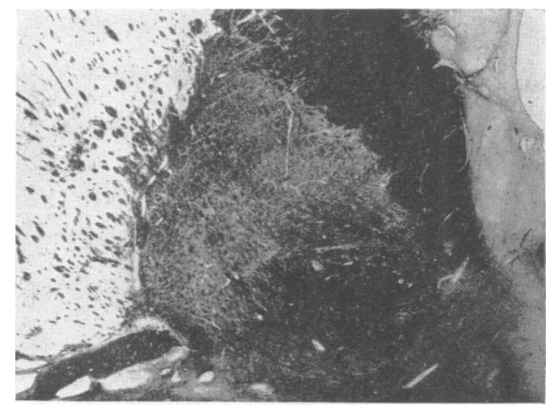

C

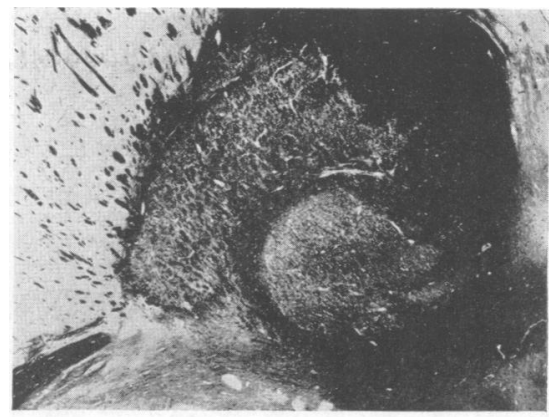

B

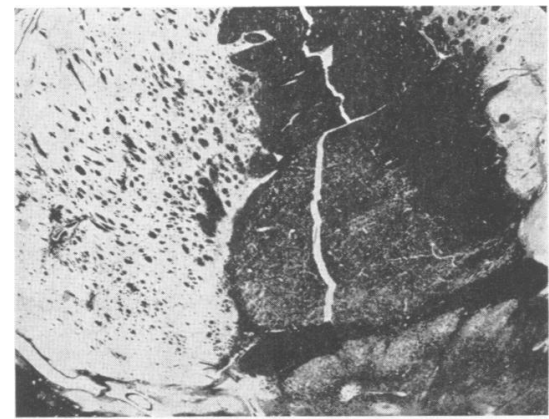

FIG. 7.-Hanc ... (Obs. III). Quatre coupes intéressant les régions pallido-luysiennes d'arrière en avant.

A. Partie caudale du Pal.: pas de raréfaction myélinique, ni des f.s.p., ni des fibres pallidales propres, par contre le C.L. présente un amincissement de son pôle latéral, de son tiers externe, de sa capsule aux niveaux dorsal et latéro-ventral. Cet aspect est typique des atrophies luysiennes secondaires. (Spielmeyer-congélation.)

B. Partie moyenne du Pal.: raréfaction marquée de g.i. et d'a.l. (Weigert-Pal-cellö̈dine.)

C. Partie caudale du tiers antérieur du Pal., raréfaction devenant plus nette en g.e. qu'en g.i., l'a.l. est même mieux imprégné. (Weigert-Pal-celloïdine.)

D. Partie orale du Pal.: la raréfaction est douteuse. (Spielmeyer-congélation.)

encore importante quoique moins touffue; une raréfaction du même ordre dans g.e., avec une gliose beaucoup plus modérée. Dans les coupes suivantes la gliose demeure encore très dense dans g.i., mais la raréfaction cellulaire n'est plus aussi extrème. Les deux autres segments demeurent inchangés. La raréfaction cellulaire est surtout nette dans la partie ventrale des trois segments. Au fur et à mesure qu'on va vers le pôle frontal du cerveau, la gliose de g.i.l. devient plus dense et les raréfactions cellulaires sont quasi uniformes (Fig. 5). Peu à peu la raréfaction cellulaire devient plus nette dans les segments g.e. et g.i.l., alors que la gliose reste encore sensiblement égale en g.i.l. et g.i.m. (Fig. 6). En avant du corps mamillaire les lésions d'éclaircissement cellulaire sont surtout visibles en g.e., cependant peu intenses, la gliose assez uniforme.

Les lésions sont donc décroissantes d'arrière en avant, atteignant en arrière surtout le segment interne et, en avant, surtout le segment externe. La gliose cellulaire est proportionnelle, ici, à la raréfaction cellulaire, mais elle demeure toujours moins importante dans le segment externe contrairement à ce qu'on voit dans les cas I et II.
Dans la série myélinique, la coupe la plus postérieure ne montre aucune raréfaction myélinique convaincante (Fig. 7A). Par contre, la pauvreté myélinique devient très nette en g.i., puis, au fur et à mesure qu'on avance vers la partie antérieure des noyaux gris centraux, oû le pallidum n'est plus représenté que par un seul segment, la raréfaction myélinique est extrèmement douteuse. C'est aussi, à ce niveau, que les cellules sont les moins raréfiées (Fig. 7B, C, D).

Nous avons repris aussi une partie de notre matériel au point de vue des lésions gliales, sans réussir mieux que la première fois à mettre en évidence une gliose fibrillaire du pallidum alors que cependant les réseaux glio-fibrillaires périvasculaires et ceux de la moléculaire sont bien impregnés: un artifice de technique peut donc, à nouveau, être exclu.

Si on envisage le processus atrophique dans son ensemble, on voit qu'il prédomine sur la partie caudale du noyau, qu'il y atteint surtout les segments g.i.m. et g.i.l. avec une gliose cellulaire proportionnelle, sans gliose fibrillaire; le segment g.e. étant altéré plus légèrement et surtout dans la région antérieure du noyau. Cette systématisation est à 
l'opposé de celle que nous avons trouvé dans nos observations I et II, où la raréfaction cellulaire la plus marquée s'observe dans les segments g.e., puis en g.m. avec une gliose cellulaire et fibrillaire plus modérée que le segment g.i. Or, si l'on compare l'évolution clinique de cas III avec celles des cas I et II les points communs sont frappants; on trouve chez Hanc . . . des paroxysmes de torsion, sur un fond de rigidité permanente. Cette hémihypertonie avec équinisme du pied s'accompagne de tremblements rythmiques des extrémités et de mouvements choréo-athétosiques. Dans ces trois cas les manifestations hypertoniques sont paroxystiques, elles sont amorcées par la mobilisation, par un stimulus sensoriel ou apparaissent spontanément, par crises. A côté d'elles existent des hypercinésies du type de la torsion axiale ou d'athétose, l'ampleur et le caractère torsionniste de ces derniers évoquent les mouvements hémiballiques. Dans nos trois cas les lésions dégénératives se limitent au pallidum, mais dans ce noyau elles ne sont pas localisées strictement aux mêmes segments. Les images myéliniques ne nous renseignent pas fidèlement sur la gravité ni sur l'étendue des altérations. Seules les images cytologiques doivent être retenues. La gliose fibrillaire peut manquer. La gliose cellulaire n'est pas nécessairement proportionnelle à la gravité de la raréfaction cellulaire. Les recherches complémentaires effectuées dans la famille Hanc. . ., depuis notre travail de 1935 , sont demeurées négatives: aucun autre cas semblable n'est apparu dans cette souche.

La superposition clinique de ces observations I, II et III est suffisament frappante pour que nous puissions, en dépit des variations d'extension et topographie des lésions dans différents segments du pallidum, considérer que ces trois observations traduisent une même hérédo-dégénérescence. Il existe, croyons-nous, une affection familiale, non congénitale, mais acquise, à évolution progressive débutant par un équinisme du pied, puis une contracture d'attitude de tout le membre inférieur, s'étendant ensuite au membre supérieur homolatéral, puis aux deux membres opposés et au cou, s'accompagnant de grimaceries et de mouvements athétosiques, parfois avec une composante ballique. A la fin de la vie on observe parfois des états d'agitation motrice extrème. Ce mélange de crises de contracture avec torsion axiale, d'hypercinésies, de spasmes de la musculature peribuccale et buccale, ne s'accompagne pas nécessairement d'une dégradation psychique. L'évolution se fait en quelques années. Il y correspond une atrophie systématisée du pallidum, bilatérale et symétrique, alors que les autres noyaux gris centraux et, en particulier, les corps de Luys demeurent indemnes.

Suivants les cas, certains segments des noyaux sont plus atteints que d'autres, mais une systématisation intranucléaire ou somatotopique, nous semble, actuellement au moins, encore indémontrable.

Cette affection est certainement indépendante de l'état pigmentaire pallido-réticulé (HallervordenSpatz), de l'état dysmyélinique (Vogt), de la paralyse agitante juvénile (Hunt) et représente une véritable atrophie systématisée et pure de l'appareil pallidal. Son diagnostic différentiel avec les deux premières maladies est, pendant la vie, délicat. D'autres observations cliniques existent, certainement, dans la littérature qui rentrent dans ce cadre; nous ne les avons pas recherchées en raison du danger d'erreur qu'il y a d'affirmer qu'il s'agit d'une affection dégénérative. Rappelons seulement une très curieuse observation de Feiling (1923) apparentée aux nôtres et qui fait toucher du doigt ces difficultés.

Dans l'observation de Feiling publiée comme "athétose double progressive" il s'agit d'un enfant de 5 ans chez qui apparaissent, après une amputation, des mouvements involontaires dans les deux membres supérieurs, puis dans le pied, dans la face avec bientôt anarthrie, des spasmes des machoires, des troubles de la déglutition. A l'âge de 9 ans, existaient des mouvements athétosiques avec hypertonie transitoire.

Nous avons suivi deux autres malades dont l'une nous a été adressée par le Dr. J. Titeca, que nous remercions ici et qui rentrent, cliniquement, dans ce groupe.

Lucette W., 22 ans (Observation IV). Le père et la mère sont polonais non-israélites. Le père a un frère bien portant avec trois enfants b.p. La mère n'a qu'un sœur, qui a deux enfants b.p. Il y a, dans la famille, quatre enfants. L'ainé est un garçon âgé de 30 ans, célibataire. La seconde est une fille qui est morte en couches d'un enfant. La troisième est la malade. La quatrième est une jeune mariée que nous avons examinée et qui est bien portante.

Histoire.-Le début remonte à l'âge de 9 ans, par une crampe à la jambe gauche. Crampe en hyperextension avec fauchage. Peu à peu, à cette crampe en hyperextension se sont combinées des secousses portant la jambe en rotation interne, puis des mouvements de flexion de la cuisse sur la jambe au moment de la marche. Environ deux ans plus tard ont commencé des mouvements brusques de flexion de la cuisse, du bassin et de rotation du bassin vers la droite. A l'âge de 15 ans apparition d'un tremblement grossier au niveau de la main gauche, avec hyperflexion de la main sur le poignet et supination forcée. Peu à peu, s'est ajouté à ce tremblement un mouvement d'écartement du bras lors de la marche. Le membre supérieur droit a commencé à présenter au niveau des doigts des mouvements athétosiques depuis environ 3 mois. La jambe droite est encore intacte. D'après les dires de sa sœur, la parole serait devenue légèrement plus scandée depuis environ un an et demi.

Examen.-Démarche grossière à la fois tremblante, cérébelleuse et dystonique. Le membre inférieur gauche est placé en extension, en varus équin et en rotation interne. Au cours de la marche il y a une hyperflexion brusque "à ressort" de la cuisse avec adduction-rotation interne en même temps bascule en avant et à droite. Les mouvements de circonduction de la cuisse vers le dedans s'accompagnent, parfois, d'un mouvement brusque de lordose avec projection des épaules en arrière, pendant la marche, le membre supérieur gauche est tenu écarté du corps à un angle de 30 à $50^{\circ}$ en hyperextension, le poignet se trouvant en hyperflexion sur l'avant-bras, tantôt en supination, tantôt en pronation, la main et les doigts étant animés d'un tremblement rythmique. Ce tremblement a la vitesse de 60 à 80 oscillations par minute. Il ne rappelle pas le tremblement parkinsonien, mais s'accompagne souvent d'hyperextension des doigts surtout de l'index comme dans les athétoses. Pendant la marche, la tête est fréquemment déviée à droite. Le membre supérieur droit présente, en ce moment, simplement, au niveau des 
articulations du poignet, de la main et des doigts de petits mouvements athétosiques en hyperextension. Dans le decubitus dorsal, les mouvements s'atténuent immédiatement, le bras gauche prend cependant l'attitude en abduction et est animé de secousses rythmiques de rotation de dedans en dehors. Le pied reste en varus équin forcé, il est souvent animé d'un frémissement. La paroi abdominale reste tendue, et cette tension empêche la recherche des reflexes cutanés abdominaux. Peu de mouvements dans le membre inférieur droit. Au cours de l'émission de la parole, de petites secousses dans la houppe du menton, le peaucier du cou surtout à gauche. Il est vraisemblable que ce sont ces spasmes de la musculature faciale inférieure qui contribuent à donner à la parole quelque chose de scandé. Les réflexes tendineux sont difficiles à obtenir à cause de la contracture. Il n'y a pas de clonus du pied, ni de la rotule. Le signe de Babinski ne peut pas être recherché à cause des mouvements involontaires. Le pied est court, massif, les doigts de pied sont étalés et boudinés. Au niveau des membres supérieurs pas de déformation des mains, pas d'atrophies, les réflexes tendineux sont conservés. Le réflexe massétérin existe normalement. Les fonctions sensitives, sensorielles et cérébelleuses, pour autant que ces dernières puissent être explorées, paraissent normales. Du côté des nerfs craniens, rien de particulier si ce n'est une parésie légère du facial droit du type central. Pas de modification du fond de l'œil. Les examens du sang et du liquide ne montrent aucune altération.

L'histoire clinique rappelle, par certains côtés, nos observations I, II, III: début dans la seconde enfance par une contracture du pied, puis du bras du même côté, avec tremblement, mouvements athétosiques et souvent des mouvements analogues à ceux décrits dans dystonies. Ces contractions brusques du bassin sur la cuisse, l'écartement forcé du bras, la déviation de la tête, contractions s'atténuant ou disparaissent dans la position couchée appartiennent en effet à la dystonie. Les mouvements alternatifs de pronation et de supination forcée des membres étendus tant supérieurs qu'inférieurs, s'apparentent aux mouvements hémiballiques. Au bout de treize ans le membre supérieur droit et la face commencent à être touchés.

L . . . M . ., 23 ans (Observation V). Parents israélites; deux enfants, pas de fausses couches. Pas de consanguinité ni d'affections nerveuses analogues dans la famille paternelle ou maternelle. Wassermann des deux parents: négatif.

Histoire.-L'enfant a été bien portant jusqu'à l'âge de 8 ans. Il a commencé alors à se plaindre de troubles de la déglutition et de la mastication. Ceux-ci dûs à des spasmes: il ne peut tantôt pas ouvrir la bouche, tantôt pas déglutir; les aliments sont projetés hors de la bouche par un spasme d'abaissement des machoires et urre protrusion brusque de la langue. En même temps ont apparu de petits mouvements involontaires des mains, qui rendent ses gestes très maladroits. A 10 ans, il présente une choréo-athétose bilatérale discrète des membres supérieurs et la démarche devient digitigrade. Nous l'avons suivi depuis 1931 jusqu'en 1940 . La parole était, au début, assourdie mais non scandée. L'intelligence paraissait intacte. La démarche devint petit à petit plus difficile à l'âge de 13 ans, elle était devenue pratiquement impossible sans soutien.

Examen, 1935.- La marche sans soutien est totalement impossible. Il peut encore manger seul, la parole n'est plus intelligible, elle est très gutturale et inarticulée. Quand l'enfant est couché et bien au repos on n'aperçoit aucun mouvement volontaire. Les pieds sont en varus équin marqué, et on note une amyotrophie légère des masses musculaires de la région antéro-externe de la jambe, du mollet, de la plante du pied et des muscles interosseux palmaires et dorsaux; il en est de même aux mains. Les mains sont, en hyperflexion sur le poignet, les doigts tendus, le pouce en abduction. Cette attitude comparable à celle des hémiplégies infantiles est assez peu variable. Quand l'enfant essaie de manger des deux mains, celles-ci gardent la même attitude. Dès qu'on parle ou dès qu'il s'émeut, les membres supérieurs s'accolent au corps en hyperpronation, les membres inférieurs s'étendent et le varus équin se renforce; en même temps la tête se met en hyperextension, les doigts, les orteils, présentent eux aussi des mouvements irréguliers d'hyperextension. Toute émotion s'accompagne d'un plissement du visage très caractéristique, la bouche se ferme comme une bourse, le menton et le massif facial se plissent, les yeux se ferment, la musculature du cou se tend. L'ouverture volontaire de la bouche est arrêtée par un spasme excessivement marqué des muscles masticateurs. Ces spasmes ne peuvent être vaincus que par l'introduction du doigt dans la bouche. Par un effort intense il parvient à déserrer les lèvres et la langue jaillit en masse hors de la bouche, comme un tube plein. Le spasme n'est vaincu que momentanément; très rapidement, les machoires se referment et le second mouvement d'ouverture est souvent plus difficile que le premier. Il ne peut plus ni rire ni pleurer spontanément. La déglutition est redevenue normale. Les mouvements oculaires sont normaux. Pas de signes pyramidaux. Conservation des réflexes abdominaux et crémastériens. Le développement psychique suit une évolution normale. Évolution sexuelle harmonieuse. Taille petite.

Examen oculaire (1931; Dr. Gallois).-Forte myopie avec astigmatisme sans troubles de l'équilibre des yeux. Pas de lésions du nerf optique ni de la rétine. Pigmentation choroïdienne anormale sans qu'il y ait de rétinite pigmentaire. Le champs visuel semble normal, même en bas. Il est difficile de dire s'il y a ou non un scotome central. En 1933 le répérage de petits objets dans une demi-obscurité était moins bon. Myopie de 5 à $7 \mathrm{~d}$. avec astigmatisme de $3 \mathrm{~d}$. Pigmentation " en carrelage" très marquée, surtout au pôle postérieur. En 1935 (Dr. Bauwens) forte myopie de $7 \mathrm{~d}$., astigmatisme de $3 \mathrm{~d}$. Le nerf optique ne montre aucune anomalie. Le fond de l'œil de la région et du pôle postérieur est du type "tabulé " fortement pigmenté mais encore normal. Ce n'est qu'en regardant à l'extrême périphérie qu'on voit de petits amas pigmentaires sur un fond moins pigmenté qui peuvent faire penser à un début de rétinite pigmentaire. En raison de l'état psychique l'examen subjectif de la vision ne montre rien de net.

Examen, 1938.-Pendant cette première période les parents ont remarqué l'amélioration de l'intellect: il a pu faire jusqu'à des additions et des multiplications de trois chiffres. On note une diminution encore très nette de la contracture des pieds en varus équin et en hyperextension au cours de la marche. Ce spasme se relâche de temps en temps et il peut faire quatre à cinq petits pas, puis le spasme le reprend et le bloque sur place les jambes ayant tendance à se croiser. Au cours de la marche, renforcement de la contracture des membres supérieurs en hyperpronation, demiflexion des poignets sur les avant-bras, hyperextension-adduction des pouces sur les doigts étendus mais allongés contre le paume. Mouvements athétosiques typiques. Quand il tâche de prendre un objet extérieur, la préhension de son propre nez, du menton, de la tête sont bonnes; celle du marteau ou d'un crayon est fortement hypermétrique avec spasmes. Forte exagération de ces spasmes intentionnels par l'émotion. Incapacité de rire. Le pleurer déclenche les mêmes plissements toniques du masque. Incapacité de lever les sourcils pour faire de gros yeux. Toute tentative d'ouverture des paupières aboutit à un spasme des orbiculaires. Ces spasmes des orbiculaires des yeux s'accompagnent assez souvent d'un plissement de l'orbiculaire des lèvres surtout au niveau des muscles canins. De temps à autre, il est obligé pour ouvrir les yeux d'abaisser du doigt la paupière 
Famille van de L . . .

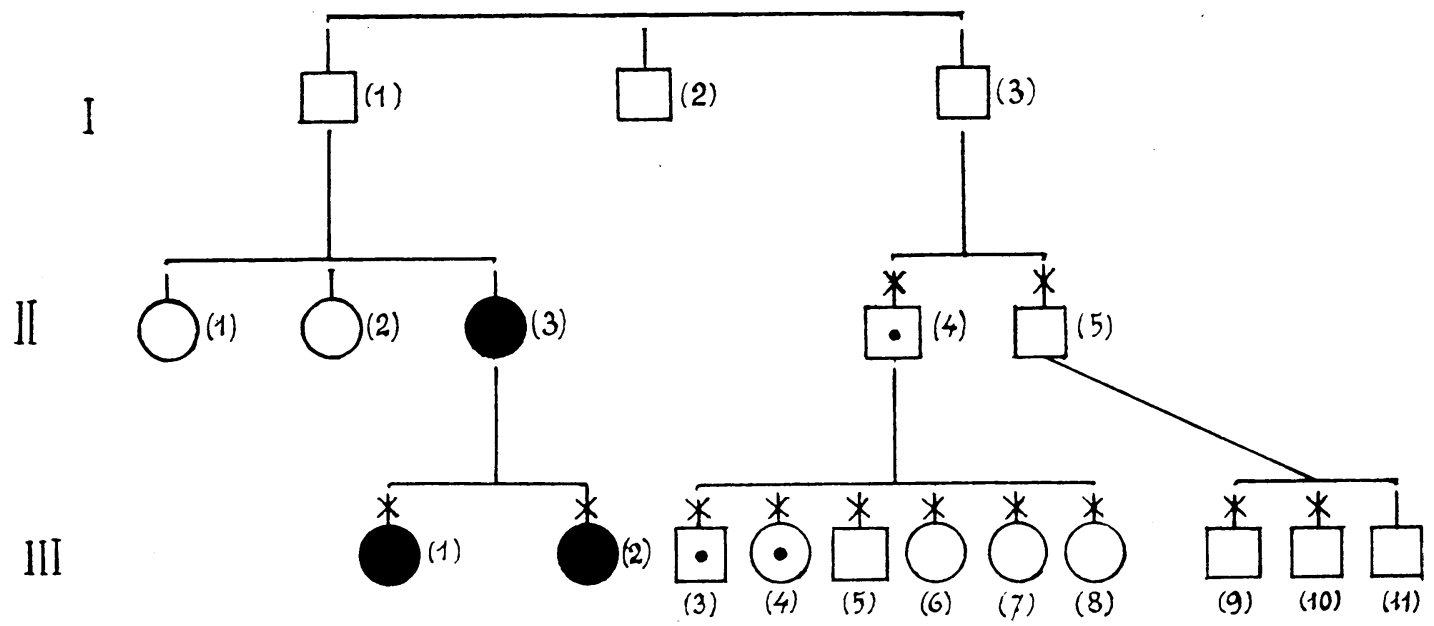

Torticolis spasmodique avec tremblement de la tête.

Tremblement de la tête seul ou accompagné d'un tremblement des membres.

inférieure. L'ouverture volontaire de la bouche est impossible; à ce moment l'enfant fait une geste agoniste consistant à pousser avec le pouce droit sur la rangée supérieure des dents en même temps qu'il éverse la tête en arrière. Ce mouvement relâche aussitôt le spasme des masticateurs. La bouche s'ouvre et la langue est projetée d'environ un quart. De temps en temps le spasme est libéré par une pression sur la machoire inférieure. Pas de troubles de déglutition. La mastication est encore possible même pour les biscottes. Toute phonation distincte est impossible, mais les siens le comprennent à certains cris gutturaux. Pas de troubles de la déglutition des liquides. Il n'y a plus jamais de rejets de nourriture. Il lit les journaux, retient, comprend toutes les plaisanteries, fait toutes les multiplications et divisions et même de petits problèmes. Pas d'aggravation des troubles visuels. Il y aurait eu pendant les premiers mois de 1939 jusqu'en avril une amélioration non douteuse. A cette époque l'enfant a fait une dacryocystite qui a beaucoup aggravé son état.

Évolution.-En 1940, les spasmes de fermeture des paupières et de la bouche étaient plus fréquents, les troubles moteurs stationnaires. Jusqu'en 1944 l'intelligence était demeurée vive; il comprenait rapidement tout ce qui se disait autour de lui et manifestait, par des gestes, cette compréhension. Il était très affectueux, particulièrement vis-à-vis de sa mère, avec laquelle il a vécu, caché en France, pendant toute la période d'occupation allemande. Il est mort d'une pneumonie grippale en décembre 1945.

La maladie s'étend ici sur une période de 14 ans et fut interrompue par une pneumonie grippale intercurrente, mais, du début à la fin, les spasmes orbiculaires faciaux et masticateurs ont été les signes dominants. Très rapidement la parole est devenue inintelligible, puis s'est établie une contracture en extension des membres inférieurs, en demiflexion, hyperpronation des membres supérieurs avec mouvements athétosiques. L'intelligence et l'affectivité demeurent normales. Il existait à la périphérie de la rétine une pigmentation anormale évoquant le début d'une rétinite pigmentaire. Les examens biologiques les plus divers sont demeurés toujours rigoureusement négatifs. $\mathrm{Si}$ nous avons rappelé ici ce cas clinique, qui est pour nous un état pigmentaire pallido-réticulé (les cas de Dercum (1925) et de Winkelman (1932) de cette affection comportaient une grave rétinite pigmentaire), c'est parce que son évolution et sa symptomatologie sont très différentes de nos observations I, II, III et IV pour se rapprocher de celles du cas de Feiling (1923), mais nous reconnaissons volontiers qu'il est impossible d'affirmer, sans contrôle anatomique, ? que les observations III et IV représentent deux maladies différentes et que l'une d'elles, seulement, appartient au groupe des atrophies pallidales systématisées.

Les observations de Dercum (1925) et de Winkelman (1932) évoquées ci-dessus furent publiées comme "dégénérescences pallidales progressives", maladie familiale autonome, mais il est reconnu aujourd'hui qu'en dépit de la préférence très étroite du processus pour le segment g.i. et l'importance des lésions neuronales, .il s'agit bien, dans ces cas, d'un état pigmentaire pallidoréticulé (Hallervorden et Spatz).

\section{L'atrophie pallido-luysienne}

Dans nos cas I et II le corps de Luys est rigoureusement intact. Dans le cas III il présente une atrophie secondaire, mais sa constitution cellulaire est respectée. Il n'en est pas de même dans l'observation VI concernant un torticolis familial et héréditaire déjà traité ailleurs (van Bogaert, 1941), à d'autres points de vue. Chez trois femmes d'une même famille, en deux générations, on trouve un torticolis spasmodique avec tremblement antagoniste à renforcement statique. Chez l'une d'elles, que nous avons suivie pendant neuf ans, le torticolis se compliquait d'une dystonie étendue à tout le membre supérieur, du côté du torticolis, avec rigidité. Elle présentait en outre des bouffées délirantes hallucinatoires et des troubles hépato- 


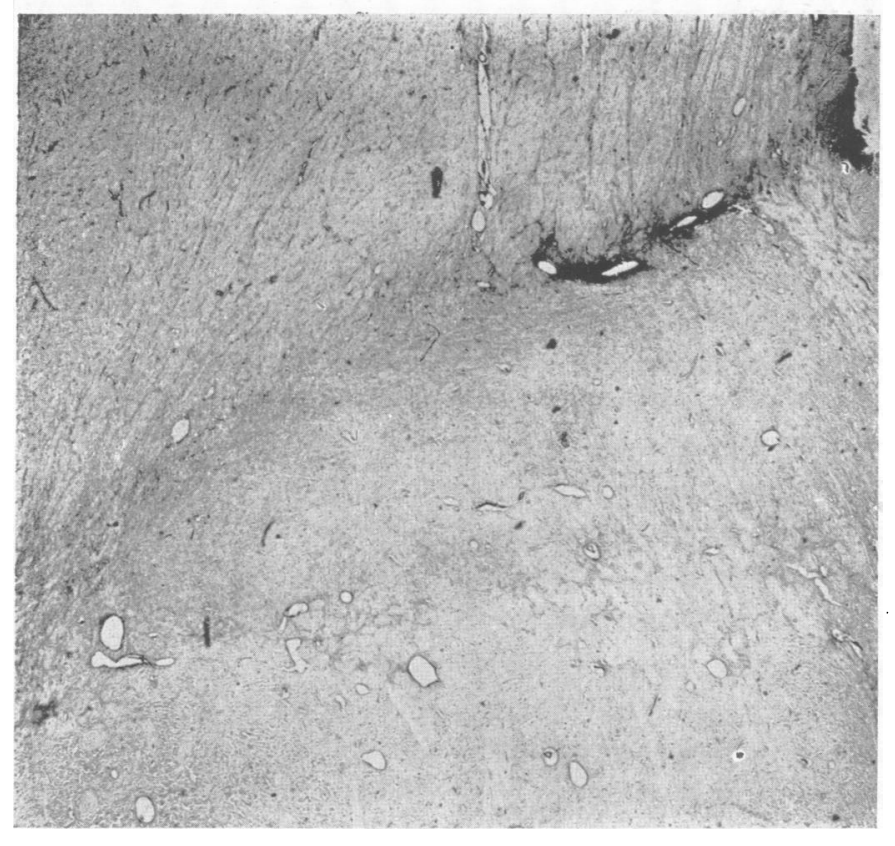

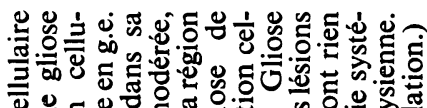

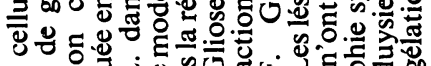

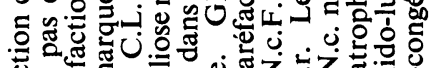
苋要

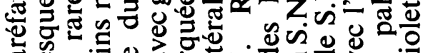

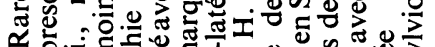
1

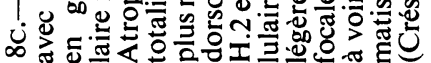
这

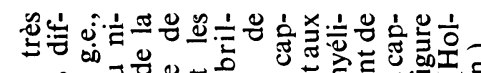

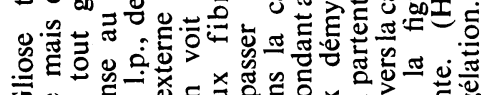

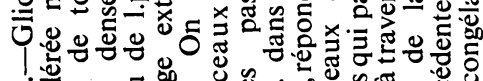

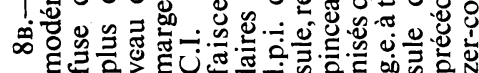
它

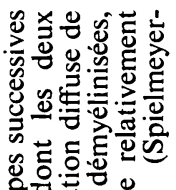

$$
\text { . }
$$
을

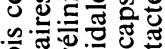
을

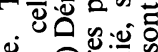

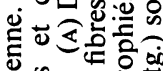
要过记 实牙

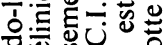

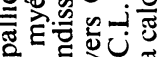
的 군

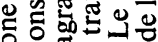
N.⿺辶

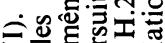

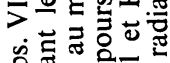
号要表一

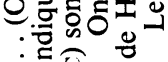

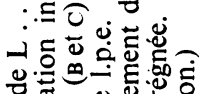
可

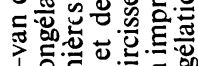

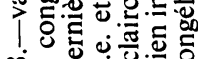
$\infty$ o. 它

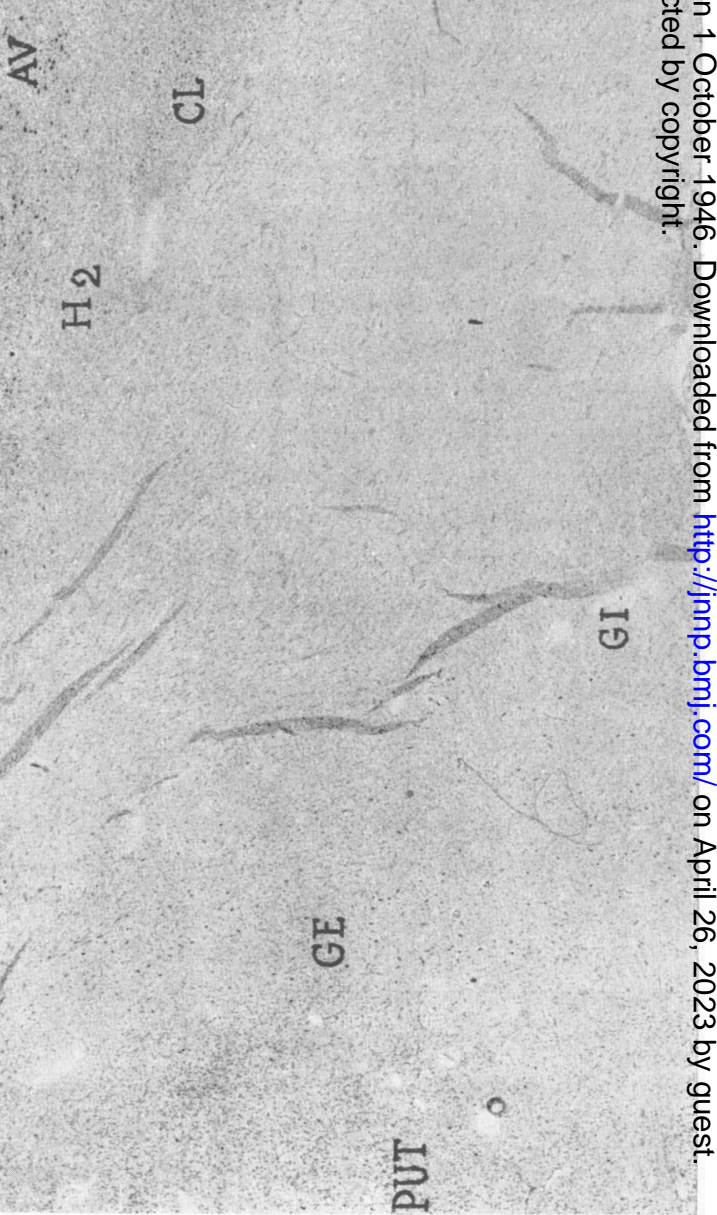


gastriques, avec des poussées de subictère. L'autopsie montra, à côté d'une augmentation de volume du foie avec de lésions banales, une gastrite atrophique.

L'examen microscopique des centres nerveux montra la coexistence d'une polio-encéphalite de Wernicke et d'une atrophie pallido-luysienne bilatérale, indépendante de la polio-encéphalite.

Nous avons réétudié ce cas sur une série discontinue (congélation: Nissl, Spielmeyer, Holzer, rouge écarlate). La coupe passant par le plus grand développement de la commissure antérieure (Fig. 9) montre un aspect grisaille de g.e., une raréfaction de l'a.l., une conservation moyenne de 1.p.i. et un aspect grisâtre des f.s.p. Les coupes cytologiques montrent une raréfaction cellulaire modérée, avec une gliose cellulaire plus marquée. Peu de glie fibrillaire. Les coupes pour les graisses indiquent une dégénérescence lipopigmentaire des cellules restantes, mais en outre une surcharge graisseuse des cellules gliales. Il y correspond, dans les Nissl, une surcharge en lipopigment et en pigment vert-noir. La coupe suivante passe par la région tubérienne et la partie antérieure de la substance innomminée: g.i. et le début de g.i.l. sont le siège d'une réaction gliale (oligo- et surtout macroglie avec de nombreuses cellules chargéęs de pigment vert, de lipopigment et d'un pigment brun-noir). Cette réaction gliale est moins nette en g.e.: à ce niveau, sauf à la partie ventrale de l.p.e., il n'y a pas de pigment décelable. La raréfaction cellulaire est modérée dans les trois segments du noyau. Peu de gliose fibrillaire. Nous n'avons malhẹreusement pas d'images myéliniques de ce niveau.

Les coupes passant par le plus grand développement du pallidum montrent un éclaircissement cellulaire très discret contrastant avec une forte gliose de g.e., une atteinte cellulaire modérée avec une gliose modérée de g.i.l., et une raréfaction extrême des cellules en g.i.m., sans presque de réaction cellulaire gliale (Fig. 10). Les préparations par la méthode de Holzer confirment cette gliose au niveau de g.i. et g.i.l. Le corps de Luys n'a presque pas subi de modifications de volume: ses cellules au niveau du tiers médioventral sont balonnées avec une gliose cellulaire dense, celles du tiers médian et dorsolatéral sont sclérosées, petites, très éclaircies et le siège d'une gliose d'une densité à peu près égale à celle du tiers médian. Les microphotographies, prises au même agrandissement que celles du cas III, montrent qu'il y a ici, dans toute l'étendue du noyau, une raréfaction cellulaire franche et non seulement une gliose. Il y a donc une atteinte primaire de ce noyau. La troisième coupe passe par la moitié antérieure du corps de Luys. Les coupes myéliniques montrent un éclaircissement net en g.i. et modéré en g.e. La coupe suivante passe par le tiers postérieur du corps de Luys et le pied, montre une pâleur uniforme de ce qui reste ici du g.i., la grisaille de 1.p.e. Les coupes cytologiques montrent

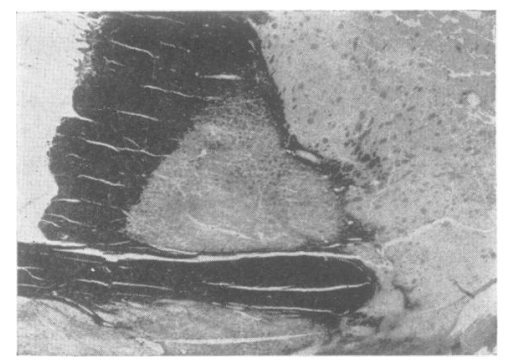

Fig. 9.-van de L ... (Obs. VI). Vue d'ensemble de lésions du Pal. oral: raréfaction myélinique marquée, éclaircissement de la l.p.e., de l'a.l. et des f.s.p. (Spielmeyercongélation.) une raréfaction modérée comme sur la coupe précédente, et les préparations gliofibrillaires montrent une très légère gliose de tout le segment particulièrement au niveau des fibres qui quittent le pallidum à travers la capsule interne et de celles qui entrent dans la composition du f.l. Le corps de Luys présente les mêmes lésions que dans ses deux tiers antérieurs. Il n'est éclairci ni dans sa capsule, ni dans ses systèmes fibrillaires propres (Fig. 8A, B, C).

Si l'on considère l'ensemble de ces lésions on est frappé par l'importance de la raréfaction myélinique de l'extrémité orale à la partie caudale du noyau, alors que la raréfaction cellulaire est modérée même en g.i. Une gliose cellulaire nette manque et celle qui existe n'est pas, en tout cas, proportionnée à la raréfaction cellulaire. La surcharge en lipopigment et pigment de désintégration beaucoup plus grande même en g.i. et g.i.l. qu'on ne le voit habituellement alors que le gliose fibrillaire fait pratiquement défaut. Il ne s'agit pas, ici non plus, d'un artéfact car dans les foyers tubériens, la glie fibrillaire est bien imprègnée. Le corps de Luys présente, lui aussi (Fig. 11), une gliose qu'on pourrait interprêter dans le sens d'une dégénérescence secondaire, mais son appareil myélinique est intact; d'autre part, les cellules montrent des signes d'altération et l'ensemble du noyau présente une raréfaction franche.

L'atrophie pallido-luysienne débutante s'exprime dans les coupes myéliniques et elle se confirme par une gliose cellulaire. Celle-ci est très modérée, contrairement à ce que l'on voit dans les observations I, II et III. Il y a un début de surcharge pigmentaire. Peut-être faut-il tenir compte de l'âge de cette malade et du fait qu'elle développe dans d'autres régions cérébrales une affection en relation avec un trouble du métabolisme, dans l'interprétation de celle-ci. La raréfaction cellulaire est marquée en g.e.; elle subit un renforcement en g.i.m. dans la partie caudale du noyau. Au torticolis clonique avec rotation de la tête à gauche, s'ajoute comme nous l'avons dit plus haut, vers la fin de la vie, une hypertonie droite avec élévation de l'épaule, avancement de la hanche amorçant une rotation axiale vers la gauche. La lésion pallido-luysienne droite n'était pas plus importante que la lésion gauche. L'altération pallidale l'emporte sur la lésion luysienne qui ne comporte pas de raréfaction myélinique.

La gravité du syndrome extrapyramidal observé chez cette malade n'est pas comparable à ce que nous avons décrit dans les cas I, II et III, où la torsion-rigidité était grossière, ainsi s'expliquent les différences qui séparent les deux tableaux histopathologiques. Cette discrétion de l'abiotrophie se retrouve aussi chez les autres membres de la famille qui présentent des torticolis à évolution lente, avec des tremblements céphaliques ou segmentaires.

\section{L'atrophie pallido-luysienne associée aux dégénérescences cérébelleuses}

Dans le cas VII le processus prédomine sur le corps de Luys à l'opposé de ce que nous avons décrit dans le cas VI et y atteint une gravité extrême. 


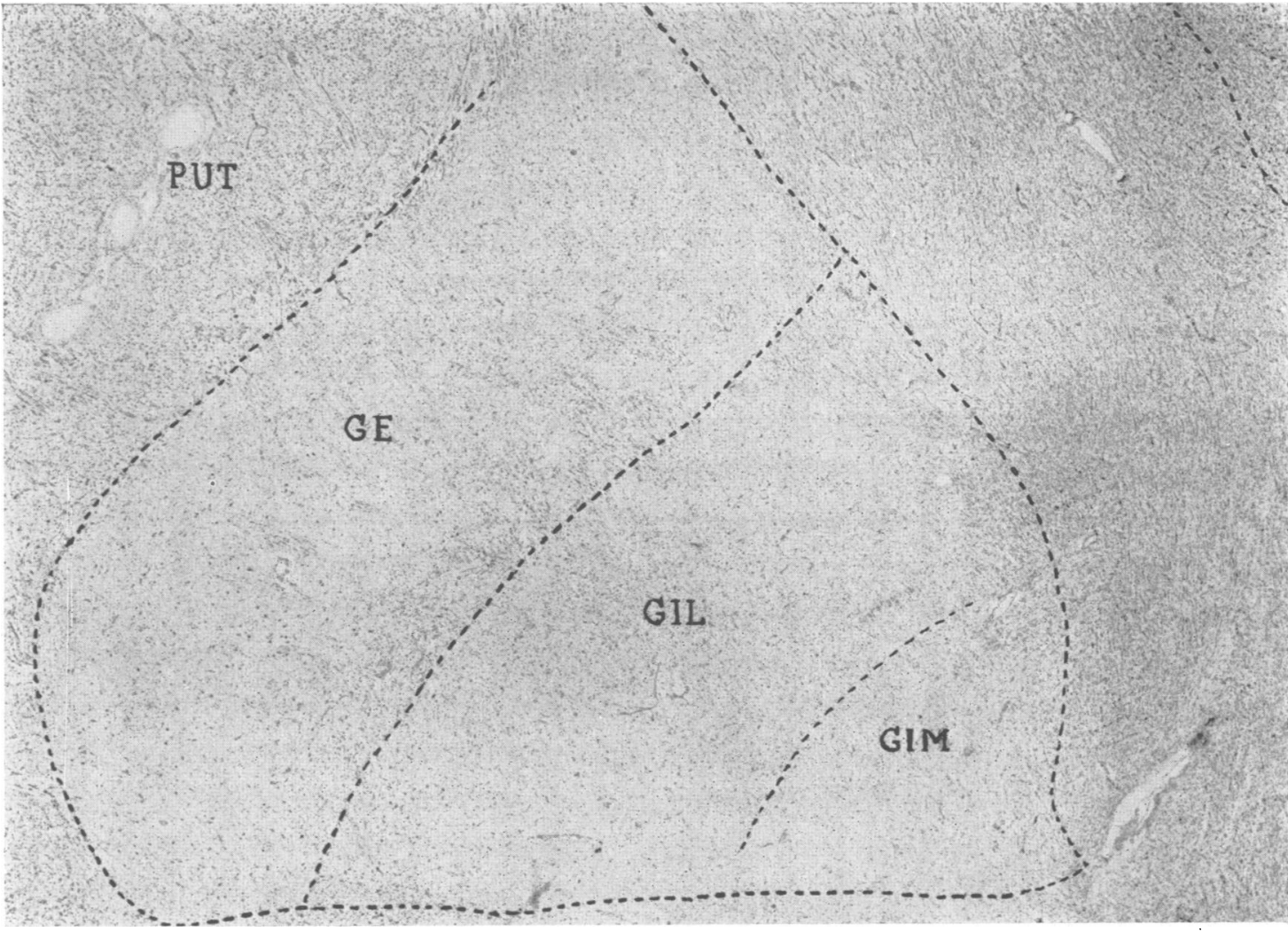

Fig. 10.-van de L ... (Obs. VI). Vue d'ensemble des lésions cellulaires dans les trois segments du Pal.: raréfaction cellulaire modérée et forte gliose en g.e., atteinte cellulaire modérée et gliose modérée en g.i.l., raréfaction cellulaire très intense avec presque pas de réaction cellulaire en g.i.m. Gliose de 1.p.i. Raréfaction cellulaire, par foyers, dans la couche optique. (Crésylviolet-celloïdine.)

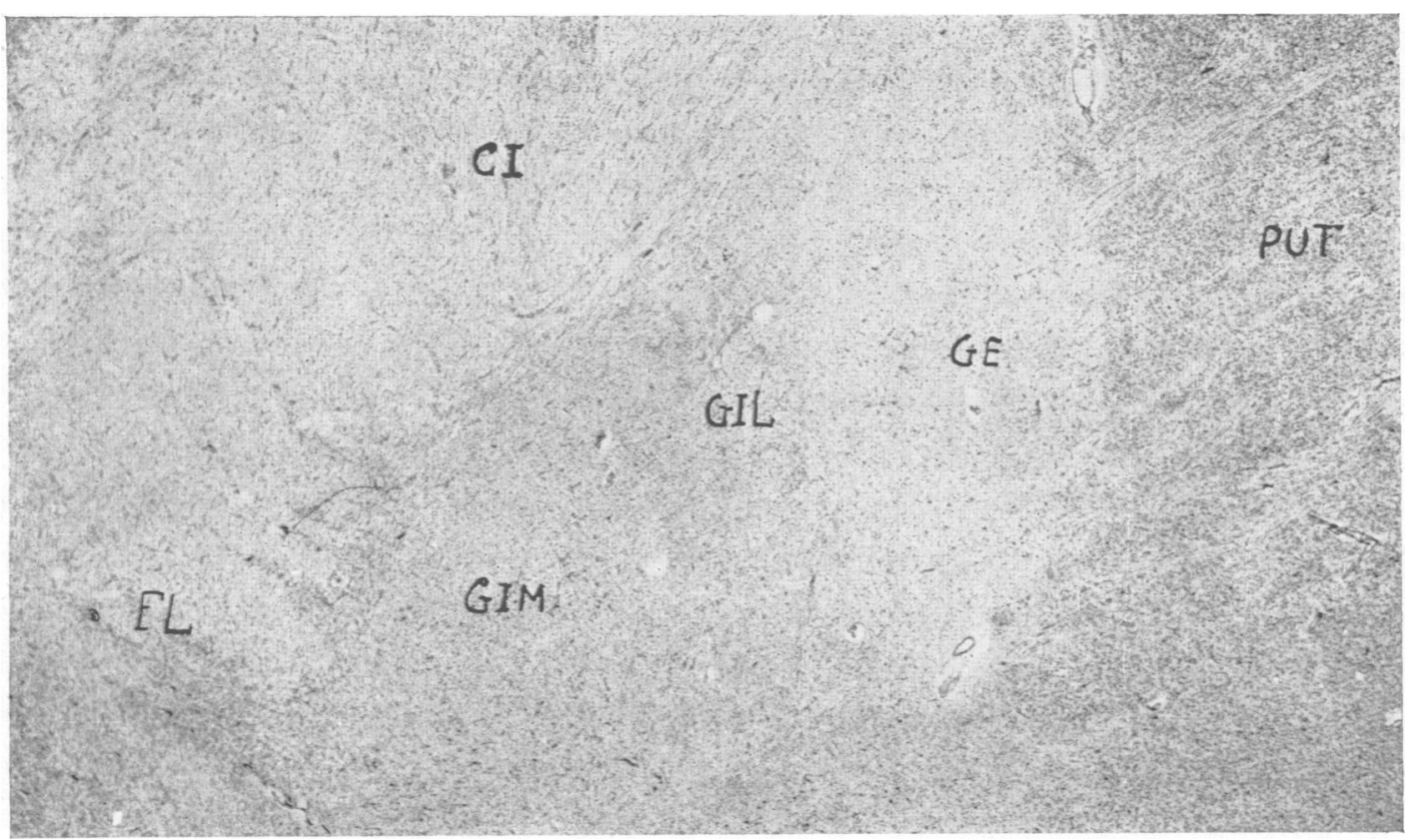

Fig. 11.-van de L . . (Obs. VI). Vue d'ensemble des lésions cellulaires dans la partie caudale du Pal. (g.e.) et la région moyenne de C.L.: raréfaction cellulaire importante en g.e., moyenne avec gliose dense en C.L. On peut suivre dans la C.I. le trajet des fibres efférentes directes du Pal. et, en H.2, celui des fibres du f.l. (Crésylviolet-celloïdine.) 
Famille Fal ...

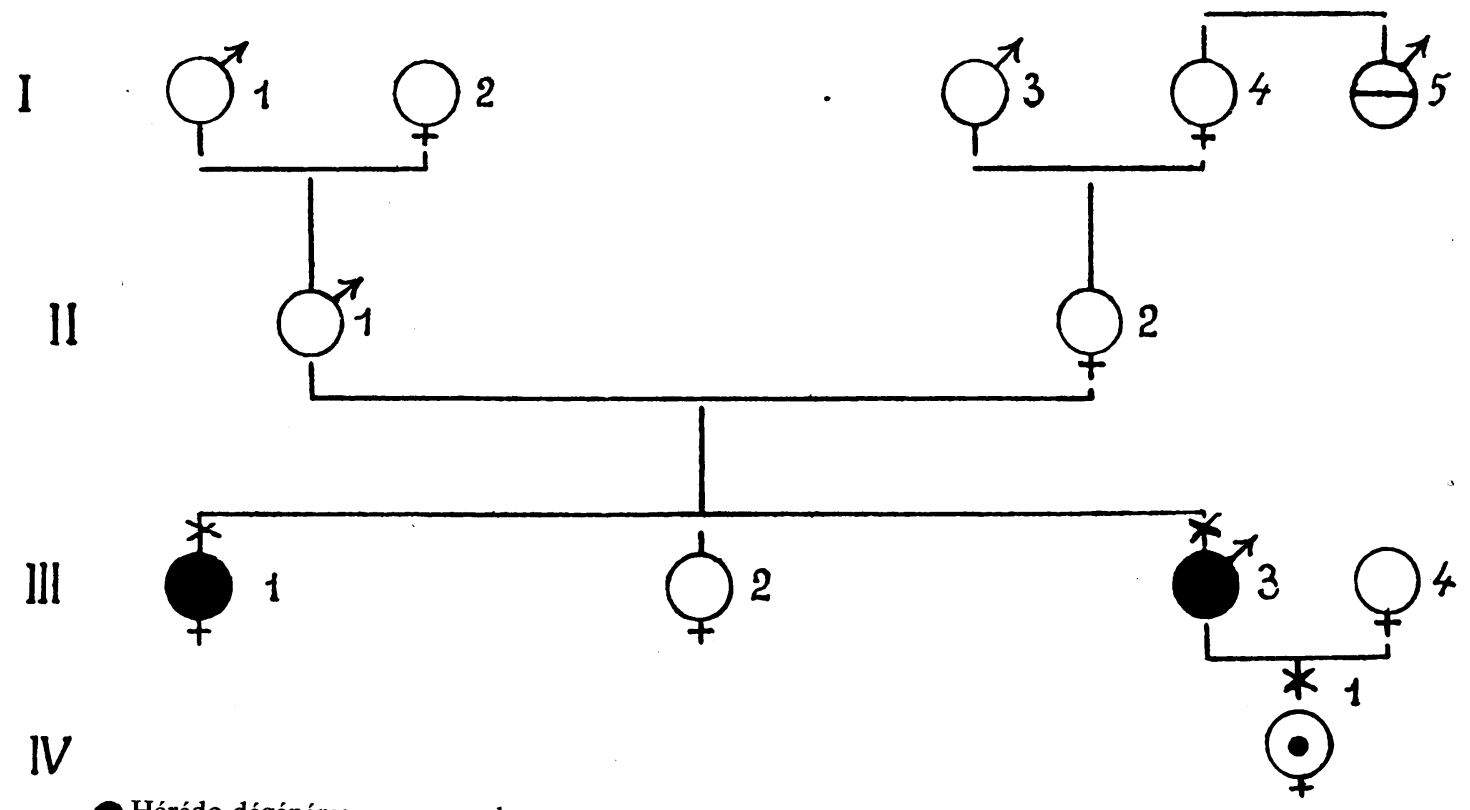

Hérédo-dégénérescence complexe avec cataracte.

Tics de la face.

$\boldsymbol{\theta}^{\top}$ Arriération mentale.

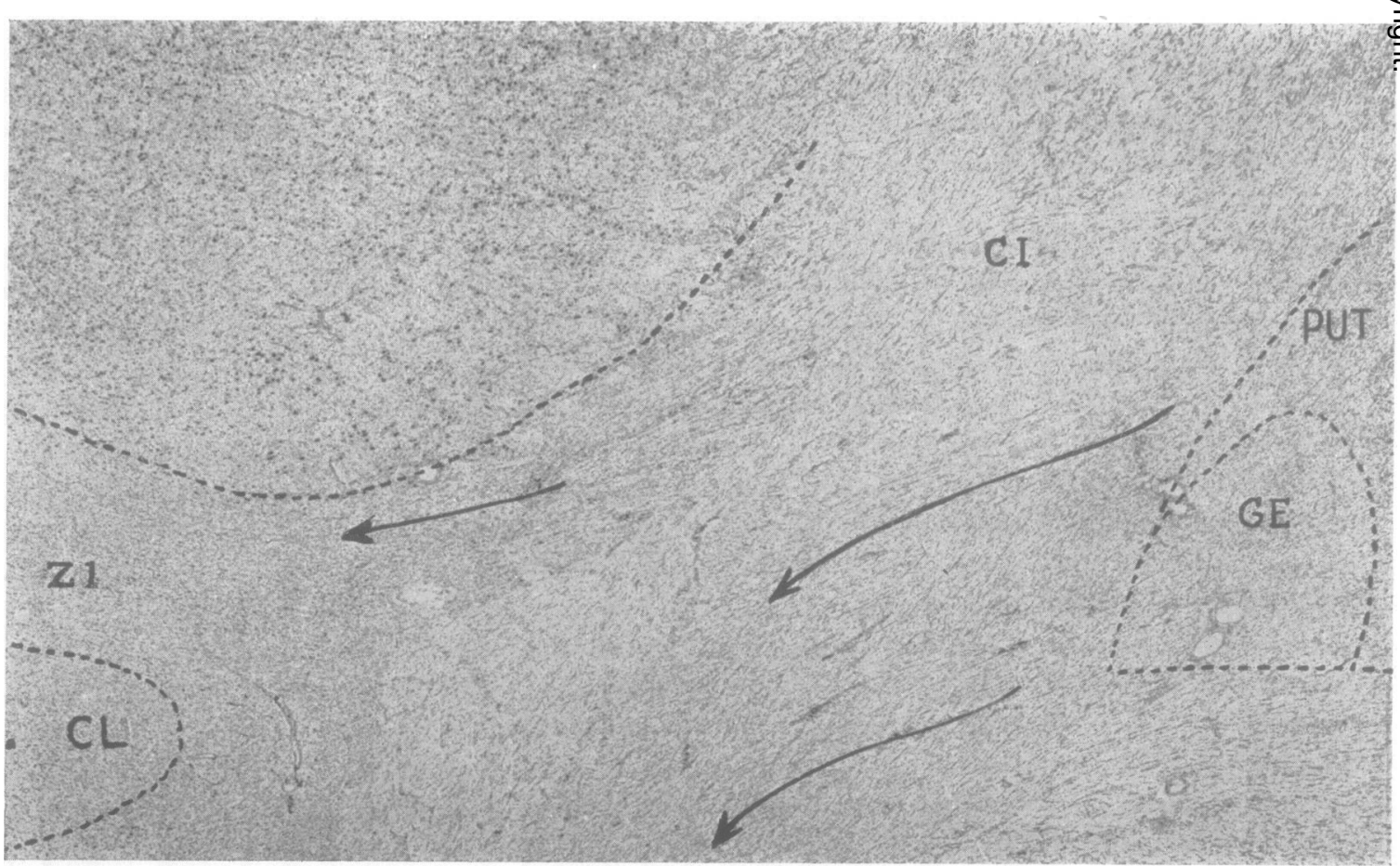

FIG. 12.-Fal ... (Obs. VII). Vue d'ensemble de lésions cellulaires dans la partie moyenne du Pal.: raréfaction marquée de g.e. avec gliose discrète beaucoup moins marquée en g.i.l.; mois encore en g.i.m. Gliose du f.l. (Crésylviolet-celloïdine.) 


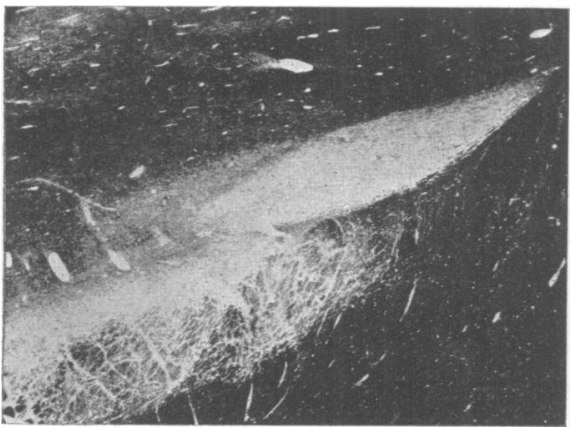

FIG. 13A.-Fal .. (Obs. VII). Atrophie extrème de C.L. à droite, prédominant sur son segment interne. Remarquer l'importance du contingent efférent vers le pied et la pâleur de H.2. (Spielmeyer-congélation.)

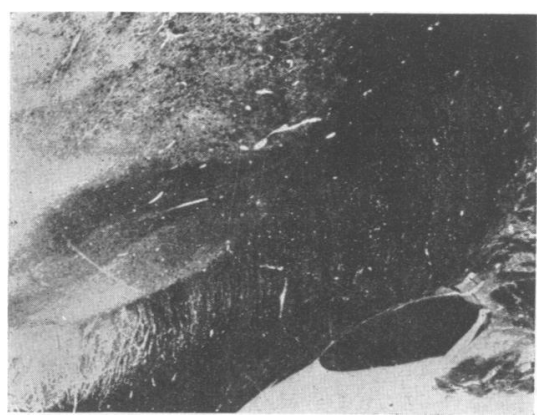

FIG. 13B.-Fal ... (Obs. VII). Atrophie débutante du C.L. à gauche: elle débute aussi dans le tiers médian, la dégénéréscence du contingent efférent vers le pied est très typique, pâleur de H.2. (Spielmeyer-congélation.)

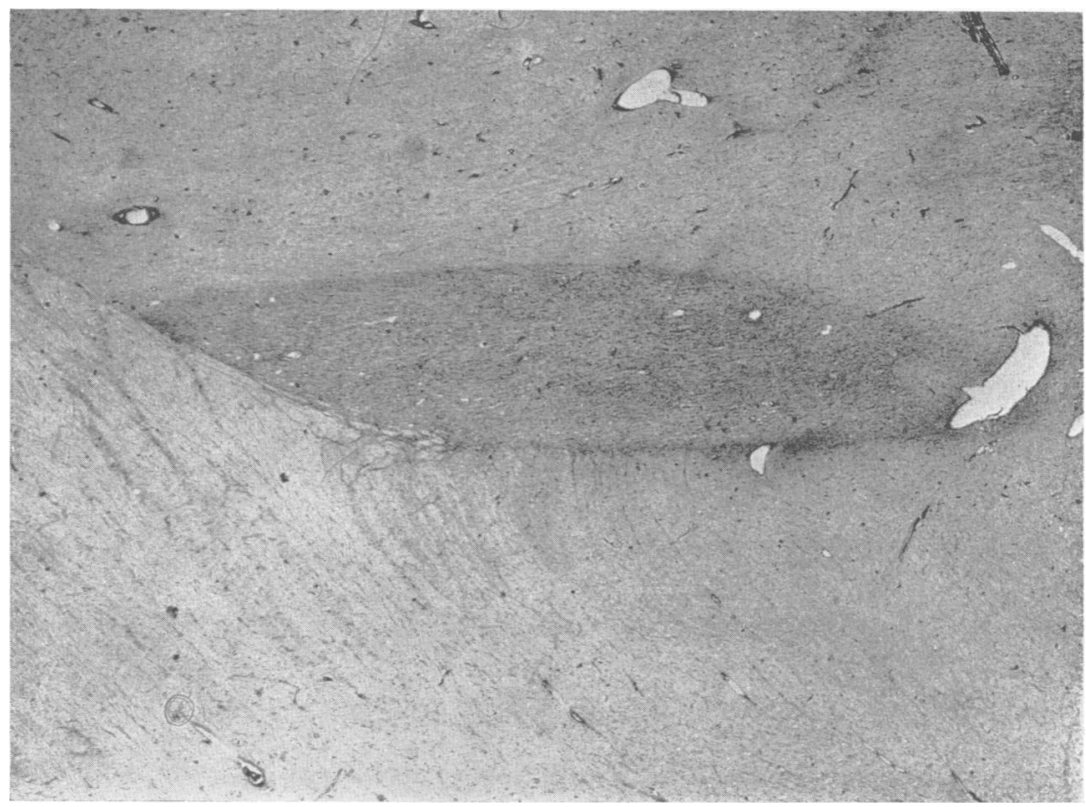

FIG. 13C.-Fal ... (Obs. VII). Gliose uniforme du C.L. renforcée au niveau de la capsule; noter la gliose des efférences vers le pied et la densité gliale uniforme du champ H.2 et H. (Holzer-congélation.)

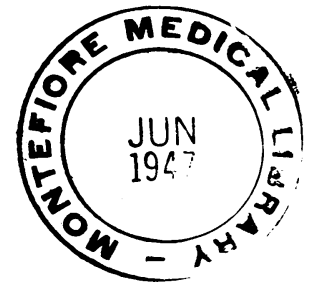




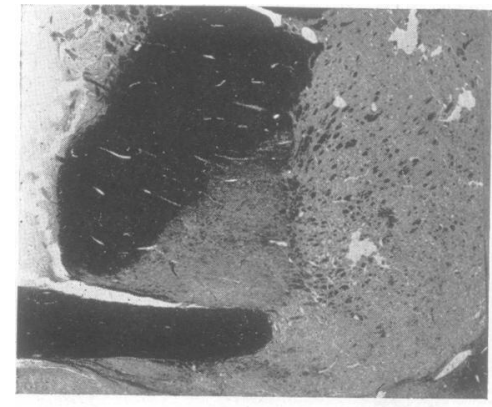

A

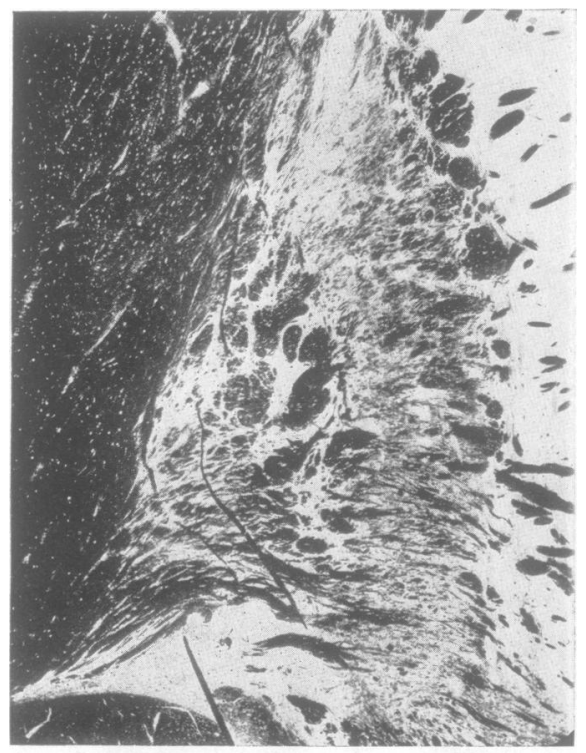

C

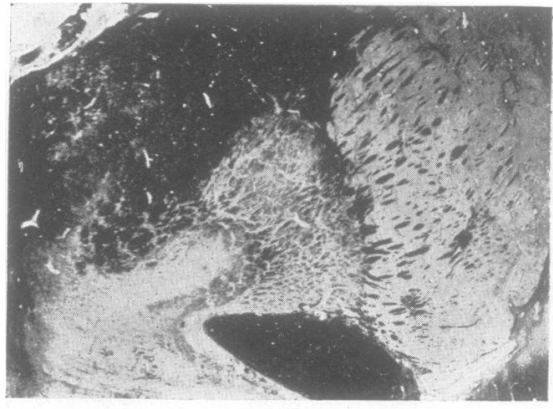

B

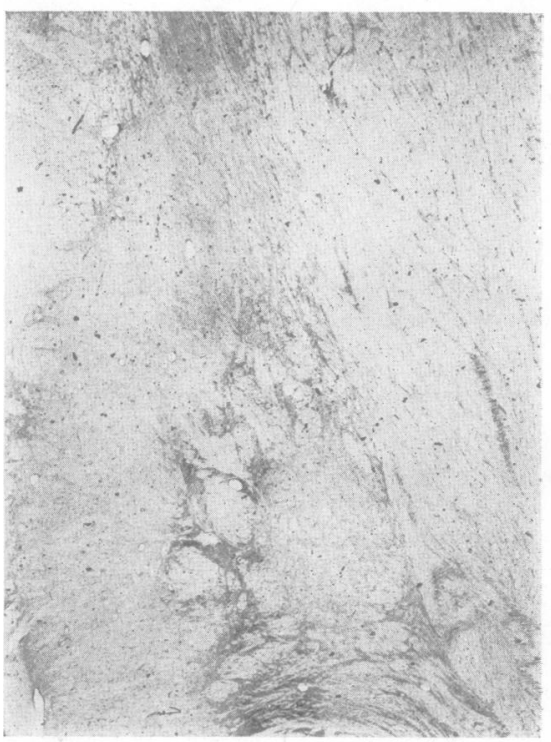

D

FIG. 14.-Fal ... (Obs. VII). Atrophie du Pal. aux différents niveaux dans le sens antéropostérieur.

A. En avant: pâleur de g.e. (Spielmeyer-congélation.)

B. Dans la partie moyenne: pâleur de g.i., pâleur moindre de g.e. sauf dans son secteur dorsal. (Spielmeyer-congélation.)

c. Dans le tiers caudal, pâleur de g.i., pâleur moindre de g.e. sauf dans son secteur dorsal. (Spielmeyer-congélation.)

D. Préparation glio-fibrillaire correspondante à la précédente: gliose dense de 1.p.i.; 1.p.e., g.i. un peu plus discrète en g.e. (Holzer-congélation.) 
Cette observation Fal ... (Observation VII) fera, incessamment, l'objet d'une publication plus complète avec notre collègue J. Titeca, qui l'a suivie cliniquement, car elle est le premier cas d'hémiballisme hérédo-dégénératif vérifié et présente, à d'autres points de vue, qui sortent du cadre de cette lecture, un grand intérêt. M. Fal ... est un homme de 44 ans, chez qui se développe, peu de temps après l'apparition d'une cataracte, une affection neurologique caractérisée par des contorsions du membre supérieur gauche, parfois de grande amplitude, et de grimaceries bilatérales: par une hypertonie du membre supérieur gauche où se déroulent les mouvements anormaux, par une démarche ataxique et une dysarthrie peu caractéristique. L'hypertonie s'étend insensiblement au membre inférieur gauche, sans déficit de fonction. Aux grands mouvements balliques s'ajoutent, mais au membre supérieur gauche seulement, des mouvements choréo-athétosiques. Aux idées délirantes du début se substitue peu à peu une démence profonde. L'étude histopathologique montre que, dans ce cas, l'atrophie pallido-luysienne est la pièce centrale d'une chaine de dégénérescences assez complexe: à côté d'une atrophie du système dentelé, qui équivaut en gravité à peu près à celle du pallidum et du corps de Luys, il existe une ébauche d'atrophie olivo-ponto-cérébelleuse avec une légère atteinte de la substance noire et des autres formations pigmentaires et une dégénérescence systématisée de cordons de Goll. Ici aussi, nous avons étudié, sur coupes sériées discontinues, les lésions de l'étage strié.

Le corps de Luys est atteint très gravement à la fois dans sa capsule, ses fibres propres et ses efférences. Il est réduit à la minceur d'une très mince lamelle biconvexe (Fig. 12) et l'effilement de sa pointe externe est saisissant si on la compare à celle d'une atrophie secondaire comme celle du cas III, par exemple. Le faisceau lenticulaire qui constitue sa partie dorso-latérale est très peu dense. Les fibres pallido-luysiennes confluant à son pôle latéral sont très réduites. L'éclaircissement du pédicule efférent, pénétrant dans le pied, est saisissant et sa dégénérescence se confirme dans les préparations gliales (Fig. 13A et C); on est surpris de voir que ces fibres naissent sur presque les trois quart ventro-latéraux du noyau et la puissance du système commissural est également impressionnante. La lésion du corps de Luys à gauche est identique, mais à son début (Fig. 13B). Le pallidum au niveau oral montre une pâleur de g.e. La coupe passant par le plein développement du noyau une disparition de la l.p.i., 1.p.e., une raréfaction des fibres propres de g.i., une légère pâleur de g.e., en tout cas moins marquée que celle du segment précédent. L'a.l. est beaucoup plus mince que normalement.

Les coupes passant par le C.L. montrent une pâleur de g.i.l., une légère atteinte moins prononcée de g.i.m. et g.e., un amincissement de 1.p.e. L'a.l. est peu développée (Fig. 14A). Le pied et le faisceau de Vicq d'Azyr ont l'aspect habituel, sauf pour les contingents pallidaux et luysiens entrant dans le pied et qui sont éclaircis. Les coupes cytologiques, au même niveau, indiquent une atteinte extrême de g.i. et g.e., de l.p.i., et un éclaircissement modéré de la partie sous-lenticulaire de la substance innominée, surtout dans ses noyaux médians. Plus en arrière la raréfaction cellulaire est plus marquée en g.e., moindre en g.i.l. et moindre encore en g.i.m., alors qu'à ces deux niveaux (g.i.l. et g.i.m.), la gliose cellulaire est très nette (Fig. 14A, B, C, D). La coupe la plus postérieure passe par le c.g.e. Les lésions de g.i. sont peu importantes (Fig. 14A). Les f.l. et f.p.l. et la partie avoisinante de g.i. sont couverts par une gliose cellulaire épaisse. La gliose fibrillaire est, en dépit de la discrétion de la raréfaction cellulaire, anormalement dense.

Cette observation VII, qui se rapproche par les mouvements torsionnistes à prédominance franche d'un côté, la contracture et les mouvements choréoathétosiques, ceux-ci strictement unilatéraux, de nos observations I, II et III, est donc essentiellement une atrophie luyso-pallidale, mais elle rentre, par les dégénérescences qui l'accompagnent, dans un autre cadre: celui des dégénérescences cérébelleuses.

La sœur de ce malade, atteinte elle aussi de cataracte acquise, se présente vers la fin de sa vie comme une ataxie cérébelleuse avec un grossier tremblement intentionnel et de troubles mentaux discrets. Or il ne s'agit pas chez Fal ... d'une atrophie cérébelleuse corticale, mais d'une atrophie olivaire, débutant au niveau des noyaux pontins et de la substance axiale, avec une très discrète atteinte corticale. Ce qui domine le tableau des dégénérescences cérébelleuses c'est l'atrophie grave du système dentelé. A côté de lui, le locus niger présente une atteinte bilatérale modérée et systématisée. Le noyau rouge lui-même ne dégénère pas. Par contre, le corps de Luys est le plus gravement altéré; son atrophie et sa dégénérescence atteignent ici un degré que nous n'avons observé nulle part dans une affection systématisée. L'atrophie atteint les deux parties du noyau: on voit, de ce chef, combien en est important le pédicule efférent qu'il jette dans le pied et l'importance de son système commissural. Le pallidum est, lui aussi, touché, surtout dans sa partie orale, où les deux segments sont totalement dégénérés. Dans la région' moyenne, l'atteinte se localise aux g.e. et g.i.1.; en arrière, g.e. et g.i. sont peu raréfiés quoique la glie cellulaire et fibrillaire y soit également dense et cela surtout en g.i. C'est à l'atteinte du pallidum qu'il faut attribuer la gliose des lames médullaires, de la capsule luysienne, du contingent dorsolatéral du pied et de l'anse lenticulaire. La lésion pallidale et luysienne prédomine à droite; elle débute, à gauche dans la partie orale du pallidum, au niveau du g.i., et dans la partie ventromédiane du corps de Luys. Le syndrome cérébelleux demeure camouflé derrière l'hémiballisme progressif avec contracture et choréo-athétose; tout au plus, la démarche à base élargie du début, pouvait elle faire penser à une affection de ce groupe, mais cette démarche se voit aussi au début de certaines chorées chroniques.

Par son aspect clinique, ce cas rentre, à l'évidence, encore dans le cadre extrapyramidal. Il n'en est plus de même dans l'observation suivante ou en raison de l'absence de toute hypercinésie ou contracture, personne n'a pu penser à une affection dégénérative des noyaux gris centraux. Une atrophie pallidale peut en eff et compliquer le tableau anatomique d'une hérédo-ataxie du type PierreMarie et y être associée à une atrophie luysienne encore beaucoup plus frappante. L'atrophie 
luysienne, soulignée pour la première fois par Greenfield (1934), se retrouve dans une de nos observations personnelles, qui sera publiée prochainement à un autre point de vue par $\mathbf{M}^{\text {me }}$ André-van Leeuwen, mais que nous évoquerons maintenant.

Dans le cas I de Greenfield (1934) les fibres strio-luysiennes sont dégénérées au niveau de leur passage dans le pied. La moitié dorsolatérale du noyau a perdu un grand nombre de ses cellules et celles qui restent sont lésées. Dans ce cas le noyau dentelé est indemne. Dans le cas II de Greenfield on retrouve des produits de dégénérescence grossiers dans le faisceau issu du corps de Luys, vers le pied. Ces fibres sont d'ailleurs raréfiées au niveau latéroventral de la capsule du noyau. Les cellules ont disparu dans la partie dorsolatérale. Elles sont moins gravement atteintes dans la région ventrolatérale, celles qui restent étant aussi dégénérées. Dans le second cas, il y avait une atrophie bilatérale du système dentelé prédominant d'un côté.

Notre observation Frey ... (Observation VIII) concerne une hérédo-ataxie du type Marie, sporadique, à debut précoce avec un grossier tremblement intentionnel, de très gros troubles de la parole et une atrophie optique du type de la névrite rétribulbaire bilatérale. L'affection débuta chez cette jeune fille à l'âge de 9 ans et elle est morte brusquement à l'âge de 20 ans. L'examen anatomique montre une dégénérescence prédominant sur la papillomaculaire des deux côtés, une atteinte des cordons de Goll et spinocérébelleux ventral, une grave dégénérescence des olives bulbaires, une atrophie cérébelleuse corticale, une très grosse atrophie des deux systèmes dentelés, une atrophie grave des deux corps de Luys et des segments externes des. pallidums.

Le strié et le pallidum ont été étudiés sur une série discontinue à congélation (Spielmeyer, crésyl, Holzer) et sur des blocs inclus en celloïdine (Weill). Les coupes myéliniques plus antérieures passent par g.e. (Fig. 17A). A ce niveau: pâleur du système fibrillaire surtout au niveau des régions ventromédianes du noyau. Quelques hypermyélinisations dans le segment dorsolatéral du putamen, mais ne dépassant pas ce qu'on voit normalement. La coupe suivante passe par la commissure antérieure et le tuber (Fig. 15 et 17B). La lame pallidale accessoire est invisible, le g.i.m. et à un moindre degré les g.i.l. sont éclaircis, la l.p.e. est bien dessinée, le g.e. est très éclairci. L'anse lenticulaire est moins dense qu'habituellement. La coupe, passant en avant du corps de Luys, montre seulement un éclaircissement égal de g.e., de g.i., et de 1.p.e. Les f.s.p., l.p.i., l.p.e., sont conservées. Le noyau g.i.l. n'est pas aussi bien impregné qu'habituellement. La coupe qui passe par la partie antérieure du corps de Luys montre une démyélinisation moins poussée et surtout nette dans les deux tiers latérodorsaux de ce noyau (Fig. 16). L'extrémité médioventrale est mieux impregnée. Le faisceau H.2 est peu net. Raréfaction uniforme de g.i. et g.e. L.p.e. et 1.p.i. sont éclaircies également. Sur celle qui passe par les corps de Luys (partie moyenne) (Fig. 17C), le g.i. est démyélinisée dans sa totalité, la g.e. est atteint un peu moins gravement, la l.p.i. est encore identifiable, 1.p.e. a son apparence normale. Le C.L. est démyélinisé dans ses fibres propres mais sa capsule est bien conservée. Le faisceau H.2 est légèrement éclairci, les f.s.p. sont assez bien marquées. Le faisceau commissural luysien et les fibres du pédoncule efférent sont éclaircis. Pâleur de g.e. surtout dans sa partie dorsale. Les coupes cytologiques montrent une raréfaction cellulaire très marquée en g.e. avec gliose. L'atteinte de g.i.l. et g.i.m. dans les coupes passent par le plein développement du pallidum est beaucoup moins importante: elle est encore plus importante dans g.i.l. que dans g.i.m. La gliose est, dans les deux segments, modérée mais indiscutable.

La raréfaction et la gliose du corps de Luys sont égales dans toutes les parties du noyau. Les raréfactions myéliniques sont donc ici diffuses quoique prédominant sur le segment externe plus nettement que dans le cas VI. La raréfaction cellulaire est en g.e. très poussée, moins profonde en g.i.m. et g.i.l. mais avec une gliose fibrillaire modérée. Le corps de Luys présente une raréfaction cellulaire très marquée, diffuse, avec, dans l'étendue du noyau, une gliose cellulaire et fibrillaire très dense et rappelle celle du cas VII. Si nous comparons entr'eux les cas VII et VIII, on voit que, pour une lésion luysienne analogue, l'atrophie pallidale est variable dans son extension intranucléaire. Dans le premier (VII) elle prédomine dans la partie orale du noyau et au niveau de g.e. et g.i.l.; dans le second, elle se poursuit dans toute son étendue antéro-postérieure et prédomine sur g.e. Dans le cas VIII les parties antérieures du noyau ventral de la couche optique présentent une gliose anormale due vraisemblablement à la dégénérescence de son contingent pallidal, et dans les deux cas on peut suivre le contingent $\mathbf{H} .2$ dans le région sousoptique sur les préparations fibrillaires.

\section{L'atrophie pallidale associée aux hérédo- dégénérescences cérébelleuses}

Si l'on considère notre observation VI comme une forme pure d'atrophie simple pallido-luysienne, nos cas VII et VIII représentent alors des atrophies pallido-luysiennes insérées dans le cadre d'une atrophie cérébelleuse tantôt corticale (VIII), tantôt olivo-ponto-cérébelleuse (VII), avec, dans les deux, une atrophie importante dentelée. On peut dès lors admettre qu'une atrophie pallidale pure (du type de nos observations I, II et III) puisse s'observer également dans l'hérédo-dégénérescence cérébelleuse. Qu'il en soit réellement ainsi ressort de l'observation Del ... (IX) ci-dessous. La famille Del ... comporte trois cas d'atrophie cérébelleuse tardive du type Marie-Foix-Alajouanine, ayant débuté vers la soixantaine, dont l'un observé par nous présente les lésions que nous allons décrire, et dont l'autre est encore vivant, âgé de 67 ans, et présente le même tableau classique. Ces deux malades étaient des alcooliques, et l'on trouve dans les noyaux du tuber et de la bandelette optique, le noyau périventriculaire juxtatrigonal et la substance innomminée de Reichert des proliférations gliales du type de celles qu'on observe dans la poliencéphalite de Wernicke. On trouve, en outre, à côté d'une atrophie cérébelleuse corticale à prédominance dorsale et vermienne une atteinte des lamelles dorsales des olives bulbaires et une atrophie pallidale débutante.

\section{Jean Del . . . (Observation IX).}

Histoire.-A fait un premier séjour, à l'âge de 34 ans, dans un hôpital psychiatrique en 1911-12, pendant 

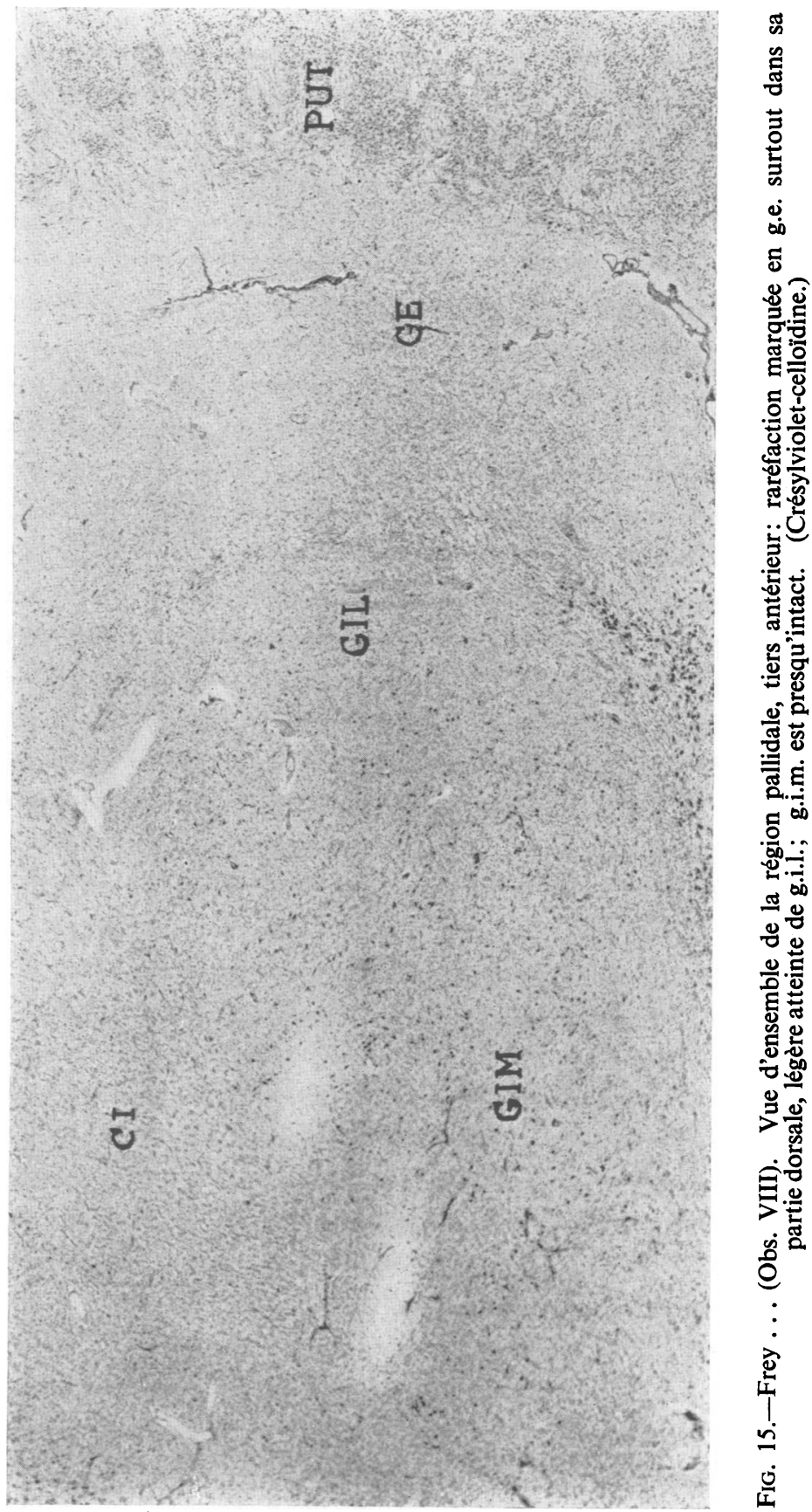


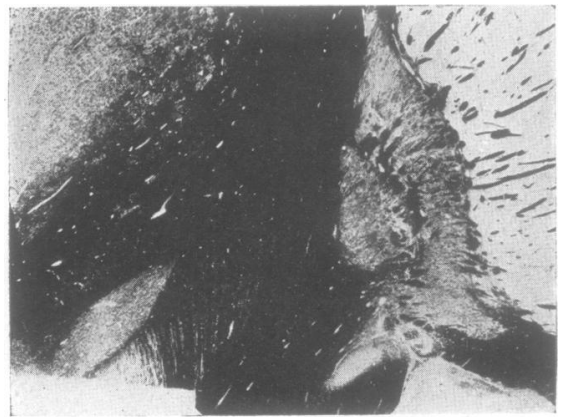

FiG. 16.

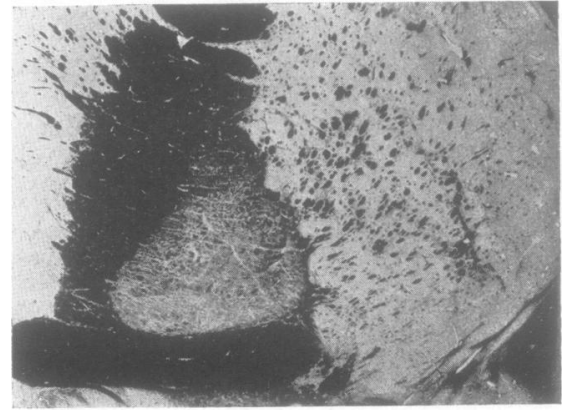

FIG. 17A.

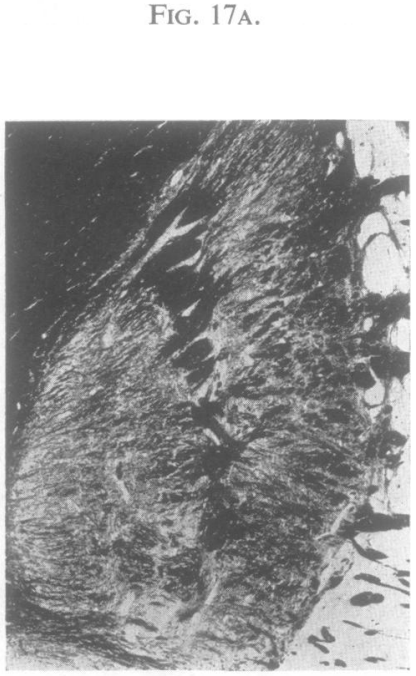

FIG. 17c.

FIG. 17B.$$
\text { FiG. } 17
$$

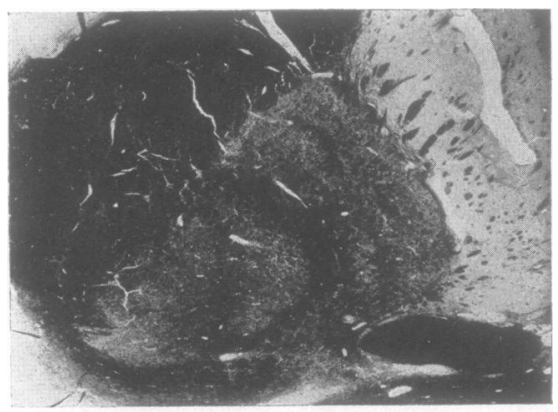

FIG. 18.

FIG. 16.-Frey .... (Obs. VIII). Vue d'ensemble de la région pallidale postérieure et luysienne; atrophie uniformê du pallidum, avec un renforcement au niveau du pôle dorsal de g.e.; atrophie importante du C.L. surtout gravedans sa moitié médiane, atrophie du faisceau papillo-maculaire systématisée. (Spielmeyer-congélation.)

Fig. 17.-Frey ... (Obs. VIII). Atrophie du Pal. aux différents niveaux dans le sens antéro-postérieur.

A. En avant: atteinte de g.e. surtout dans la région ventro-médiane. (Spielmeyer-congélation.)

B. Dans le tiers antérieur: pâleur de g.i.m., g.i.l., l.p.m. et un peu moins de g.e. (Spielmeyer-congélation.)

c. Dans le tiers postérieur: pâleur uniforme et modérée de g.e., g.i.l., un peu plus poussée de g.i.m.; effacęo ment de la 1.p.i. (Spielmeyer-congélation.)

Fig. 18.-Del ... (Obs. IX). Tiers moyen du Pal. L.p.m. et 1.p.i. sont reconnaissables; g.e. présente une modifica tion moniliforme de ses fibres propres d'où son aspect granité, l.p.e. est éclaircie, les f.s.p. sont pauvres. (Spielmeyer-congélation.) 
six mois, pour un épisode maniaque. Depuis cette époque a séjourneé, chaque année, à l'hôpital de Schoten, pendant six à huit semaines, pour un état mental comportant des angoisses et des hallucinations visuelles. C'est un alcoolique invétéré depuis l'âge de 28 ans. Il n'y aurait pas eu de polynévrite. En 1917 a fait un séjour d'un mois à Gheel. Les premiers troubles de la marche ont apparu à l'âge de 58 ans; ils se sont aggravés surtout depuis quatre ans.

Examen (1941).-Démarche ataxique et spasmodique avec élargissement de la base de sustentation. Grossière dysmétrie dans l'épreuve du doigt sur le nez et du talon sur le genou surtout à droite. Dans l'attitude debout il oscille d'avant en arrière et la tête est animée d'un tremblement ample, plus lent que le tremblement sénile ou congénital (50 à 60 oscillations par minute). Assis, les membres supérieurs sont souvent atteints de mouvements alternatifs de pronation et de supination, discontinus; ils sont entretenus dans toute position d'équilibre instable. Il s'y ajoute parfois de petits mouvements reptatoires des mains comme dans une chorée de Sydenham. Les réflexes tendineux sont vifs; les réflexes oculaires conservés et normaux. Ataxie oculaire. Pas de nystagmus. Fond d'œil: artères scléreuses. Pouls veineux spontané. Au point de vue mental: grosse désorientation dans le temps et dans l'espace, fausses reconnaissances. Rares hallucinations visuelles.

Évolution.-La marche est devenue impossible depuis le début de 1944, chute en arrière, grave dysmétrie des membres supérieurs. Parole sourde mais encore intelligible. Décédé le 26 mars 1945.

Le tableau clinique est celui d'une atrophie cérébelleuse tardive classique sauf le tremblement de la tête, plus marqué qu'habituellement, et les petits mouvements reptatoires des mains assez curieux. Les troubles mentaux pouvaient être attribués aux excès alcooliques. Nous ne reprendrons de notre protocole histopathologique que ce qui concerne les noyaux gris centraux (Fig. 18, 19A, B, C, D).

Le Pal. montre au niveau d'une coupe passant par la partie postérieure de la C.a. une pâleur de g.e. avec un aspect moniliforme des fibres propres. L.p.i. et 1.p.a. sont bien conservées, l.p.e. est éclaircie. Les fibres strio-pallidales (f.s.p.) sont peu denses et peu impregnées. Le Put, est traversé dans le sens ventrodorsal par de grands vaisseaux dilatés. La 1.p.i. est coupée par quelques lacunes. Les coupes cellulaires montrent, dans la partie orale, mais surtout dans le tiers antérieur et. moyen, un éclaircissement cellulaire très net de g.e.: les cellules restantes sont transformées en vésicules graisseuses, la glie elle-même est chargée de granulations grasses. Pas de gliose fibrillaire. Les segments g.i.l. et g.i.m. sont le siège d'une simple gliose cellulaire sans graves pertes cellulaires et sans gliose fibrillaire. Le Put. présente un certain nombre de petites criblûres au voisinage desquelles les grands et petits éléments sont raréfiés. La coupe passant par la partie caudale du noyau montre une bonne conservation de g.i. avec gliose cellulaire, une gliose fibrillaire des fibres striopallidales qui traversent le g.i. Le C.L. est intact au point de vue cellulaire et myélinique: il présente une simple gliose cellulaire.

L'atrophie du segment externe du pallidum s'observe donc, ici, dans toute l'étendue du noyau, sans gliose fibrillaire et avec un éclaircissement myélinique. La raréfaction cellulaire est la plus marquée à partir du tiers moyen jusqu'en arrière. Les proliférations cellulaires gliales sont, à certains niveaux, proportionnelles. Le putamen n'est pas tout à fait intact; les fibres strio-pallidales sont moins bien impregnées que normalement; mais les altérations striées ne sont ni quantitativement ni qualitativement comparables à celles du pallidum. Le corps de Luys montre une gliose secondaire très modérée. L'importance des lésions du segment externe du pallidum est moindre que dans le cas VIII; les cellules restantes présentent une dégénérescence graisseuse qui n'épargne pas la glie cellulaire néoformée. Cette raréfaction des cellules pallidales dépasse considérablement ce que nous avons observé dans les cerveaux témoins du même âge et doit être considérée comme pathologique. L'observation IX ne présente sans doute qu'un exemple particulier de l'extension striée des lésions dans une dégénérescence cérébelleuse et se rapproche, par là, entr'autres, d'une observation de Scherer (1933), où une importante atteinte striopallidale accompagne le tableau d'une atrophie olivo-ponto-cérébelleuse (cas F.A. 1879 de son mémoire).

Nous avons rapporté ici l'observation IX à cause du caractère strictement localisé de l'atrophie

Famille Del ...

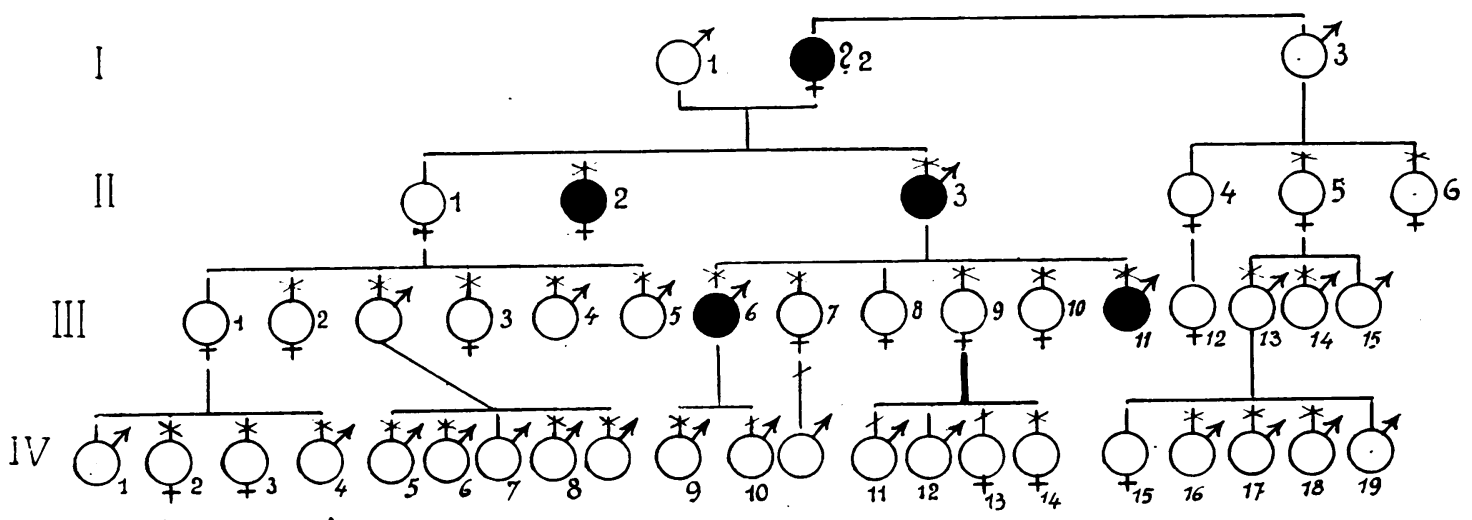

Atrophie cérébelleuse tardive, type Marie-Foix-Alajouanine. 


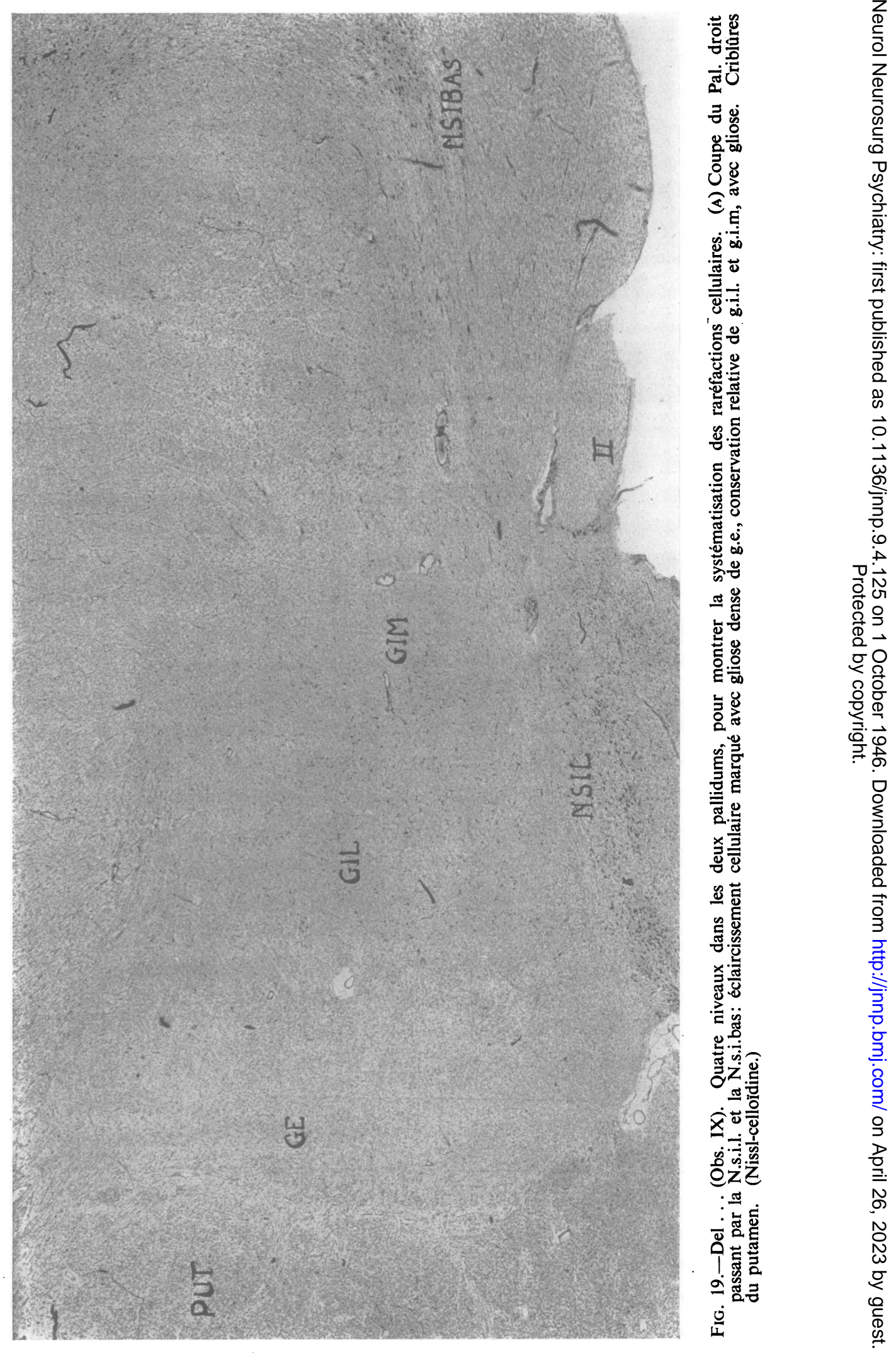




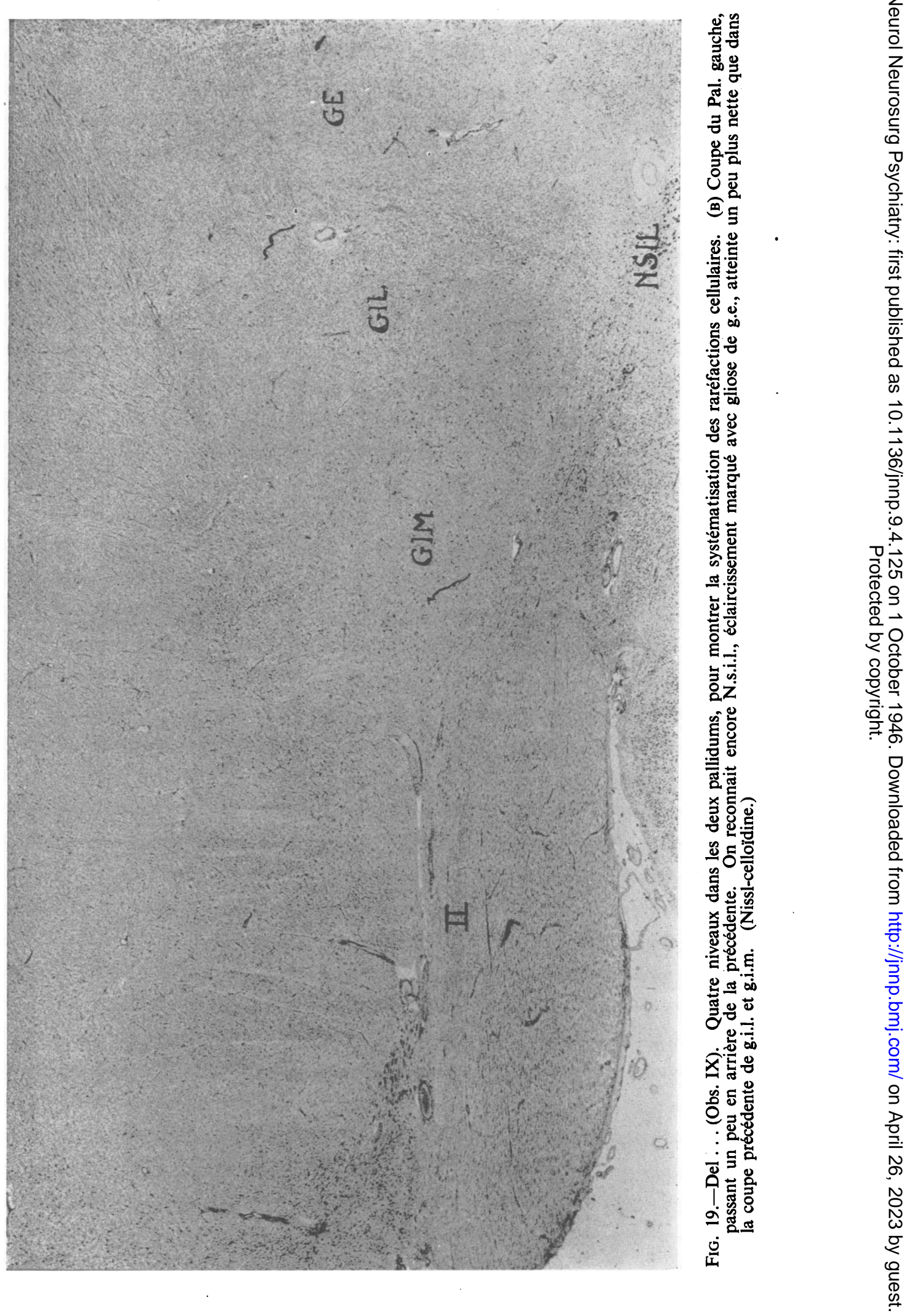



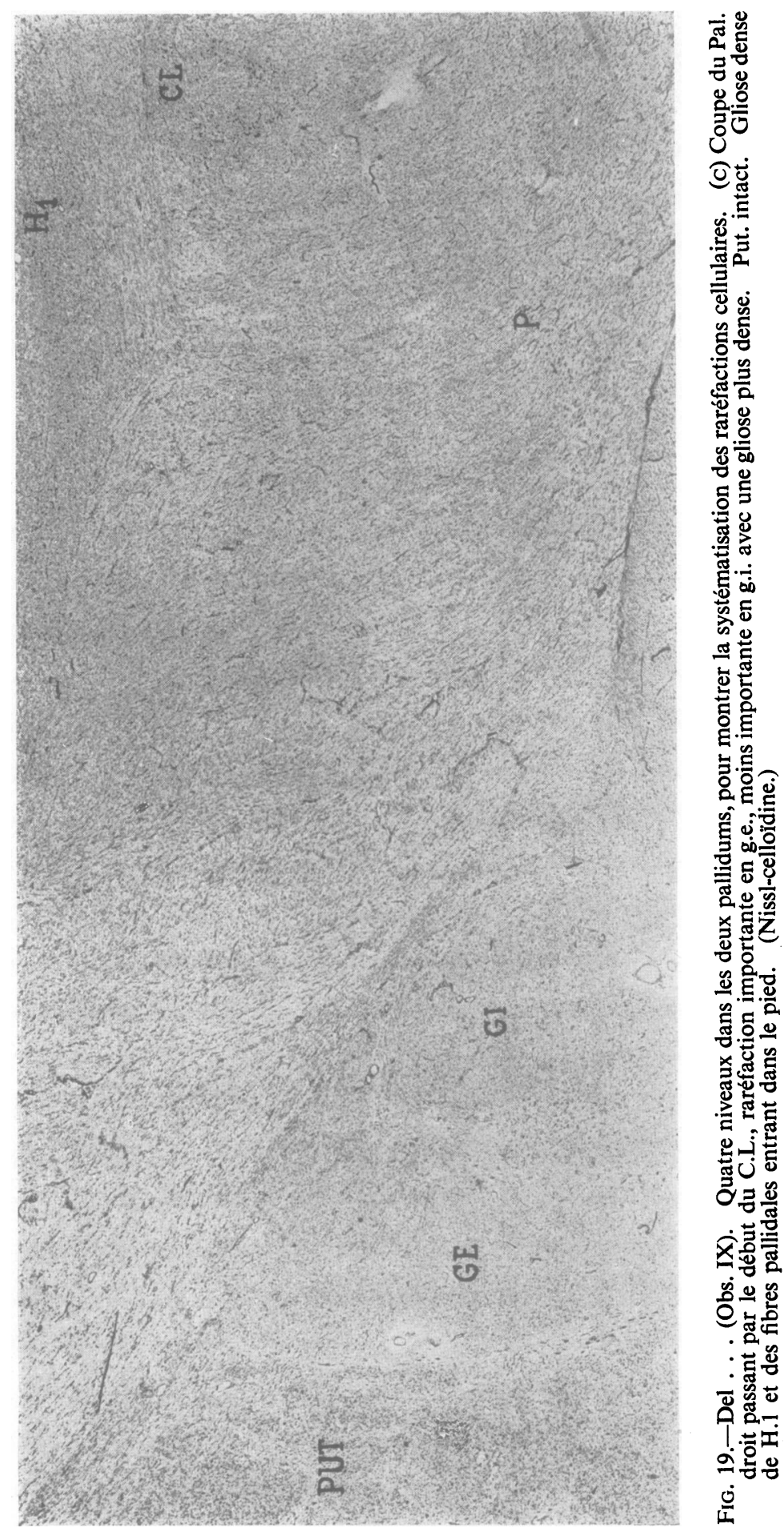


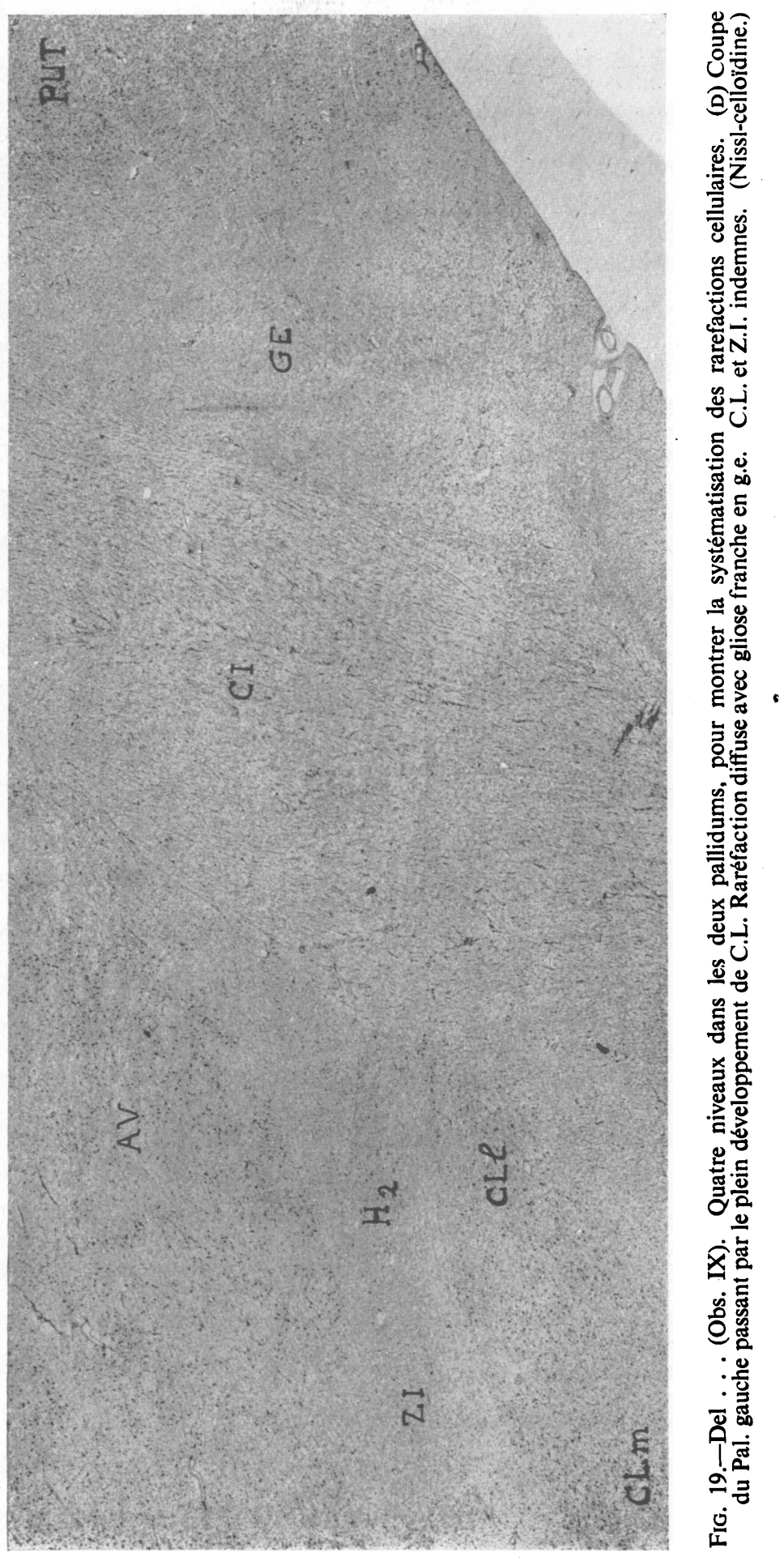

$\stackrel{-1}{+}$

N

品

율 운

$\therefore$

잉

웅

융 흘 के

뭉 
du segment pallidal externe, alors que le strié et le locus niger sont conservés.

\section{Commentaires}

De la juxtaposition de cette série de cas, sans doute très polymorphes, mais qui ont comme dénominateur commun: l'atrophie totale ou partielle du noyau pallidal, lésion progressive, ayant les caractéristiques histologiques d'une abiotrophie, lésion apparue au cours de maladies dégénératives systématisées, le plus souvent familiales, se dégagent ces quelques notions:

\section{A. Au point de Vue Clinique}

1. Quand l'atrophie pallidale ou pallido-luysienne se produit au cours d'une dégénérescence cérébelleuse elle ne se démasque par aucun de ces signes que nous somme convenus d'appeler extrapyramidaux et rien ne permet de séparer, du vivant du malade, ces cas des hérédo-dégénérescences classiques et banales. La dégénérescence cérébelleuse annule les manifestations cliniques de l'atrophie pallidale ou pallido-luysienne. Cette notion de l'effet " négativant" des lésions cérébelleuses sur les manifestations des lésions striées ne doit cependant pas être généralisée, car nous savons qu'à certaines atrophies olivo-ponto-cérébelleuses peuvent, au bout d'une longue évolution, se substituer des syndromes rigides et akinétiques (Ley, 1925; Guillain, 1926), que des atrophies cérébelleuses familiales peuvent se muer en des états hypertoniques avec spasmes buccopharyngés (van Bogaert, 1946). Dans le premier cas, c'est à l'invasion strio-nigrique par le processus hérédo-dégénératif qu'est due la modification de l'aspect clinique (Scherer, 1933); dans le second, la modification de l'aspect clinique est due à ce fait qu'à l'altération cérébelleuse se juxtapose un état pigmentaire pallido-réticulé: dans les deux éventualités la lésion striée " escamote" alors la lésion cérébelleuse et la sémiologie striée passe à l'avant-plan. D'autre part, l'absence de phénomènes cérébelleux aussi bien qu'extrapyramidaux dans une combinaison de lésions cérébelleuses avec un état marbré du strié se trouve illustrée par une remarquable observation d'un idiot publiée par Norman (1940).

2. Quand l'atrophie pallidale et pallido-luysienne est pure ou qu'elle domine quantitativement d'une façon très marquée sur le processus cérébelleux, elle s'exprime par un mélange de contractures, de mouvements de torsion autour de l'axe du corps ou autour de l'axe des segments des membres, sur lequel se greffent des mouvements choréo-athétosiques ou des tremblements. L'ampleur des hypercinésies et des contractures dépend de la gravité et de l'extension de la dégénérescence: elle va du torticolis tremblant avec une rigidité ébauchée unilatérale (observation VI) à un syndrome de grande torsion axiale (observation III) en passant par la contracture avec hémiballisme important (observation VIII).

3. Il n'est pas indispensable pour qu'apparaissent les décharges para- ou hémi-balliques typiques que le corps de Luys soit gravement dégénéré. Celles-ci peuvent apparaître au cours d'une atrophie pallidale pure. Toutefois dans notre observation où l'atrophie luysienne domine tout le tableau, ces mouvements ont été, du début à la fin de l'évolution, le signe le plus frappant. Si donc, la libération du corps de Luys à l'égard du pallidum (par la lésion de ce dernier) suffit à les faire apparaître par moments, la lésion du corps de Luys même les déclanche d'une manière plus régulière et plus durable.

4. Ces syndromes demeurent pendant longtemps unilatéraux, sauf en ce qui concerne les désordres mimiques d'emblée symétriques, alors que les différences dans la gravité des lésions de l'atrophie pallido-luysienne ne sont en général, d'un côté à l'autre, pas très importantes. Il est fréquent de voir, dans une même souche ou fratrie, les symptômes commencer du même côté.

5. Dans une famille dont le système cérébelloluyso-pallidal est fragile, suivant que la dissolution débute ou prédomine au niveau du complexe pallido-luysien ou au niveau du complexe cérébellodentelé, ce seront les aspects extrapyramidaux, hérédo-ataxiques ou cérébello-myocloniques qui seront à l'avant-plan, mais il n'est pas exclu de les voir être juxtaposés: le syndrome extrapyramidal existe chez le frère, et, chez la sœur (notre observation VII), le syndrome cérébelleux. La sœur de nos deux frères morts d'un syndrome pallidal progressif présentait un Friedreich fruste et fixé actuellement (1946) encore dans son évolution. Ceci rappelle les rares cas de juxtaposition, dans une même famille, d'une chorée chronique et d'une hérédoataxie. Le fait qu'on retrouve, à l'étude de ces cas, à côté de l'atrophie pallido-luysienne, des atrophies incomplètes de l'appareil cérébelleux indique, avec certitude, que la maladie extrapyramidale ne doit pas sa naissance à une mutation dans le gène pathologique, mais au renforcement individuel de la dégénérescence au niveau de certains maillons du complexe neuronal familialement fragile.

6. Le syndrome d'atrophie pallidale ou pallidoluysienne comporte une association de contractures, de mouvements choréo-athétosiques, de torsions, de tremblements, d'hémiballismes, à debut segmentaires, puis dimidiés, puis bilatéraux, avec des grimaceries mimiques dès le début symétriques (si elles existent). Les spasmes de la musculature buccopharyngée sont très rares. Dans l'état pigmentaire pallidoréticulé, au contraire, les spasmes orbiculaires des yeux, des muscles péribuccaux, masticateurs, même des muscles de la déglutition et de la phonation, sont souvent, à l'avant-plan, dans la sémiologie et l'anamnèse. Les mouvements choréo-athétosiques et la rigidité y sont d'emblée bilatéraux, la rigidité y est souvent une rigidité en extension ou en flexion des membres inférieurs, les états de torsion et les ballismes y sont moins fréquents. On y signale, comme dans notre cas $\mathrm{V}$, des rétinites pigmentaires. 
Un diagnostic différentiel de ces deux formes d'atteinte pallidale peut donc être envisagé. Seule la première est une affection systématisée. Toutes deux sont des affections acquises; c'est-à-dire, survenues dans la deuxième enfance, les périodes prépubérale, pubérale ou pendant l'adolescence, et cela les sépare des états dysmyéliniques-états résiduels, dont la sémiologie fait souvent d'états de rigidité peut, toutefois, être extrèmement voisine de celle des atrophies.

Sur le point de savoir si l'état pigmentaire pallido-réticulé est une abiotrophie vraie, comme nous l'avons écrit antérieurement (1936), abiotrophie du pallidum et de la partie réticulée de la S.N.--il faut être plus réservé que nous ne l'étions, depuis que la publication de nouveaux cas (Eicke, 1940) montre que les lésions débordent sur d'autres régions cérébrales.

\section{B. Au Point de VUe systématiQue et PathologiQue}

1. Sur les coupes du pallidum et du corps de Luys traitées par les méthodes myéliniques seules on ne peut pas poser de conclusions. Tantôt les raréfactions sont tellement discrètes qu'on n'ose pas les affirmer (g.e. des observations I et II), alors que les lésions cellulaires sont importantes. Tantôt au contraire, le segment semble éclairci (g.i. dans II), alors que les altérations cellulaires y sont moins accentuées que dans le segment voisin. Il peut arriver cependant que les deux séries d'altérations (myélinique et cellulaire) soient non douteuses et qu'elles coincident dans le même segment. Dans le cas VI la raréfaction cellulaire du C.L. est importante et les images myéliniques, tout en n'étant pas normales, n'auraient pas emporté notre conviction. Dans le cas III, par contre, où il s'agit d'une simple atrophie secondaire, l'éclaircissement du pôle latéral du C.L. attire immédiatement l'attention. Des constatations analogues ont été faites dans l'état pigmentaire pallido-réticulé ou l'imprégnation myélinique parait dans certains cas presque normale; alors que dans d'autres cas les cellules paraissent normalement conservées, cela se soit aussi dans la paralysie agitante juvénile où pour une démyélinisation à peine décelable, les lésions cellulaires peuvent être importantes.

2. Il n'y a pas de rapports proportionnels entre l'intensité de la gliose cellulaire et le degré de raréfaction des cellules ganglionnaires. La raréfaction peut-être avancée (g.e. dans III) alors que la gliose cellulaire est beaucoup plus dense dans le segment voisin (g.i.), qui est moins atteint. Elle peut être modérée pour une raréfaction poussée (dans le cas II en g.e.; chez VI; dans g.i.m. et g.i.l. chez VII, etc.). Elle peut être nette alors qu'il n'y a pas de raréfaction cellulaire appréciable (dans g.i.l. et g.i.m. du cas IX).

3. Il y a plus fréquemment un rapport proportionnel entre la gliose fibrillaire et la raréfaction des cellules ganglionnaires (par exemple, chez I et II), mais cette règle n'est pas non plus absolue. La raréfaction cellulaire peut prédominer sur un segment (g.e., cas VI) alors que la gliose fibrillaire prédomine sur le segment voisin (g.i.). La gliose fibrillaire peut faire entièrement défaut alors que la réaction gliale cellulaire est nette et qu'on ne peut pas incriminer une faute de technique (cas III).

4. Il n'y a pas, avec nos techniques, de rapport proportionnel entre les degrès de la gliose cellulaire et fibrillaire; les exemples invoqués ci-dessus permettent de ne pas y insister. Pour le corps de Luys, les glioses cellulaires et fibrillaires ne sont pas non plus proportionnelles à l'importance de la raréfaction cellulaire. Les glioses cellulaire et fibrillaire de la dégénérescence secondaire (par exemple, dans l'observation III) peuvent être importantes et contraster avec l'intégrité cellulaire. Dans d'autres cas, comme VIII, où la raréfaction cellulaire est extrème, la gliose cellulaire est minime, la gliose fibrillaire n'est pas plus dense que dans des cas (comme VII) où la raréfaction ganglionnaire est moins grossière.

5. Dans aucun de ces cas, sauf en VI et là encore dans la partie antérieure seulement de g.e. et de g.i.l.-et d'une manière modérée-nous n'avons trouvé de dépôts anormaux de pigments sidérophiles, de pigments verts et de graisses. Dans le cas IX seul, nous avons noté une dégénérescence graisseuse des cellules pallidales et d'un certain nombre de cellules macrogliales hors de proportion avec l'âge du sujet.

6. L'extension des lésions, à l'intérieur du pallidum, n'est dans aucun de nos cas limitée à un segment de ce noyau. Peu intense et diffuse dans le sens antéro-postérieur dans le cas IX, elle est un peu plus accentuée et également diffuse dans le cas VIII. Dans les cas I, II et VII, elle est surtout franche dans les deux tiers antérieurs du noyau. Dans le cas II il y a une prédilection pour la région ventromédiane; dans le cas III, pour les régions ventrales. Dans la plupart des cas, le segment externe (g.e.) est de beaucoup plus gravement atteint au point de vue cellulaire (I, II, VII, VIII et IX); il peut arriver qu'il le soit autant que le segment interne (g.i.) (cas III). Dans un seul cas (VI) les lésions prédominent dans le segment interne (g.i.).

7. L'extension des lésions dans le corps de Luys est assez uniforme dans le sens antéro-postérieur. Sauf dans le cas VI, où le tiers médian est moins gravement atteint, elle est également uniforme dans le sens transversal. Dans les cas VII et VIII la raréfaction est diffuse, quelques cellules échappent aux pointes et au bord ventral et médian de ce noyau.

Nous avons, bien entendu, tenu compte dans ces appréciations du fait que normalement le segment g.e. du pallidum nous parait moins riche en cellules à cause de la minceur de celles-ci que le segment g.i.; que la moitié médiane du C.L. a des cellules plus petites et plus claires que la moitié latérale et que la gliose y est plus dense.

Ces faits notés pour le pallidum et pour le C.L. éliminent d'emblée, dans nos cas, toute possibilité de considération sur les répartitions somatotopiques 
des lésions, discussion que l'évolution même de nos cas rendrait d'ailleurs vaine, sauf peut-être l'observation VI.

8. Quant aux relations entr'elles des différentes atrophies, il faut envisager séparément celle du Pal. et C.L., puis celle de ces deux noyaux et des éléments cérébelleux. Une atrophie secondaire luysienne peut s'observer dans l'état dysmyélinique et dans l'état marbré, mais pas nécessairement. La lésion secondaire peut se borner à une gliose fibrillaire et cellulaire avec éclaircissement des fibres efférentes du pallidum comme dans notre observation III.

Nous admettrons que dans certains cas (par exemple, notre observation VI) ce retentissement puisse aboutir à l'atrophie du noyau lui-même par une dégénérescence transsynaptique, à condition que la lésion pallidale soit suffisamment importante. Les cas VI et VII satisfont à la seconde condition mais pas tout à fait à la première étant donné que la raréfaction cellulaire y est moins avancée dans le Pal. que dans le C.L. Que cette atrophie transneuronale ou transsynaptique du C.L. est bien facultative, l'intégrité de ce noyau dans les cas I, II et III avec lésion pallidale importante est là pour le démontrer. Quant aux liens qui relient l'atrophie pallido-luysienne et celle du système cérébelleux nous avons cru, un moment, que l'atrophie importante du noyau dentelé pouvait y jouer un rôle. On sait que ce noyau envoie des fibres au noyau latéral ventral de la couche optique, et que le noyau antérieur ventral de la couche optique reçoit des fibres du pallidum. Malheureusement ces noyaux ne sont pas grossièrement altérés dans nos coupes. D'ailleurs le cas IX, où le p.c.s. est rigoureusement intact, montre que cette interprétation doit être abandonnée. Nous considérons donc les atrophies pallido-luysiennes et cérébelleuses toutes deux comme primaires; c'est-à-dire, comme non subordonnées ou, si l'on préfère, coordonnées.

$\mathrm{Au}$ point de vue hodographique, nos cas ne permettent que des conclusions réservées car leur survie a été trop longue pour qu'on puisse suivre les voies au fil des corps granuleux ou osmiophiles et les lésions des centres ne sont pas parcellaires. Néanmoins si l'importance des apports pallidaux dans la composition de l'a.l. et du f.l. ressort de nos cas, ou les lésions prédominent souvent sur le g.i., est en accord avec les notions acquises actuellement; les contingents pallido-luysiens qui n'empruntent pas le champs $H$. et venant soit du g.i. soit de g.e. (Ranson, 1943) nous ont paru spécialement fournis. Nous n'avons pas pu mettre en évidence, avec certitude, le contingent pallidohypothalamique; par contre, nous observons une gliose légère de la S.N.r., de la partie orale du N.R. et du N.tg.l., alors que le C.L. dans cette observation II est intact. Une afférence pallidale vers ces formations est donc probable quoique peu fournie, et ceci confirmerait l'opinion des Mettler (1942). Sur l'aboutissement du faisceau H.1 nous n'avons rien de spécial à ajouter sauf qu'il vaudrait la peine d'étudier plus systématiquement que nous n'avons pu le faire ici les lésions thalamiques qui accompagnent l'altération pallidale.

Les travaux expérimentaux les plus récents sur le singe (Glees, 1945) montrent les connexions très étroites du pallidum avec le noyau ventral antérieur, avec le noyau latéral de la C.O. et avec le noyau ventromédian hypothalamique. Un certain nombre de fibres après avoir croisé dorsalement le C.L. se dispersent entre le C.L. et le corps mamillaire jusqu'au noyau interpédonculaire. Tout document manque à ce subjet chez l'homme. Nous nous proposons de reprendre plus tard l'étude de nos cas sur ce point.

\section{Au point de vue physiopathologiQue}

$\grave{A}$ ce point de vue nous voulons demeurer des plus prudents, et nous en avons apporté, dans notre introduction, les motifs. La lecture de la pathologie expérimentale chez le singe depuis les premiers travaux de Wilson (1913) jusqu'aux plus récents des Mettler (1942) et de Kennard (1944) ne jette malheureusement pas encore de lumière sur ce que l'on observe à l'échelle humaine. Les expériences de destruction bilatérale du pallidum chez le singe (trois cas) par Ranson et Berry (1941) ne provoquent, chez cet animal, ni tremblement, ni choréoathétose, ni roue dentée: c'est-à-dire, aucun des signes evoquant le syndrome pallidal humain. Dans un très curieux cas de sidérose pallidale chez $M$. rhésus Glees (1943) n'observe aucun signe clinique or, cet état se rapproche étrangement de l'état pigmentaire d'Hallervorden-Spatz, non seulement par la présence des mêmes pigments mais par celle des lésions graves des fibres et des cellules pallidales. On trouvera dans un excellent exposé d'esprit neurochirurgical de Meyers (1942) une revue critique de nos connaissances physiologiques, pathologiques et pathogéniques, sur les tremblements alternatifs, les rigidités et la festination démontrant suffisamment "les obscurités que les chercheurs cliniciens rencontrent en face de la physiologie du complexe extrapyramidal ".

Nous n'avons voulu réunir ici que quelques faits anatomo-cliniques étudiés sous un certain angle, étude dont nous sommes les premiers à reconnaître les lacunes. Comme neurologiste, on doit se contenter de décrire aussi minutieusement que possible cette sémiologie fluide et diverse et d'en acter les équivalents histopathologiques. Une question générale peut cependant se poser, à propos de ces mouvements, touchant à leur physiopathologie. Faut-il les considérer comme l'expression d'une " lésion déchargeante" (discharging lesion) de Jackson des éléments altérés; notion dont l'intérêt a été brillamment renouvelé récemment ici même (Martin, 1945), ou comme une "overaction" de centres libérés. Martin souligne lui-même que les symptomes des décharges spontanées sont plus explosifs, plus spasmodiques, plus forcés, et qu'ils se produisent indépendamment de l'état de veille ou de sommeil, alors que ceux qui résultent d'une "overaction" des centres inférieurs sont plus continus et moins brutaux et qu'ils disparaissent pendant le sommeil. C'est de cette seconde catégorie que se rapprochent les symptômes décrits plus 
haut. Nous avons précisé la localisation des noyaux lésés, mais laissé dans l'ombre, délibérément, toute considération sur la nature et le rôle des noyaux libérés.

L'étude des maladies extrapyramidales entrera peut-être dans une nouvelle phase maintenant que la physiologie expérimentale s'attaque à nouveau à l'étage strio-pallidal avec des méthodes plus raffinées. De leur côté, la clinique et, sur ses pas, l'anatomie pathologique n'ont pas de raisons de ne pas tenter d'apporter pierre à l'œuvre commune, mais elles ne peuvent qu'essayer de " situer " les questions à résoudre. Mieux qu'à n'importe quel chapitre de la neurologie, on peut appliquer à celui-ci une phrase de Claude Bernard citée par Charcot dans sa Leçon d'ouverture célèbre: "Il ne faut pas subordonner la pathologie à la physiologie. C'est l'inverse qu'il faut faire. Il faut poser d'abord le problème médical tel qu'il est donné par l'observation de la maladie, puis chercher à fournir l'explication physiologique: agir autrement, serait s'exposer à perdre le malade de vue et à défigurer la maladie."

\section{BibliographIE}

Alcock, N. S. (1936). Brain, 59, 376.

Bielschowsky, M. (1919). Jhrb. Psychiat. Neurol., 25,1. Bouché, G., et van Bogaert, L. (1935). Rev. neurol., 64, 886.

Brockhaus, H. (1938). Jhrb. Psychiat. Neurol., 49, 1. (1942). Ibid., 51, 1.

Charcot, J. M. (1925). Leçon d'ouverture reproduite dans Paris méd., 1, 465.

Davison, C., Goodhart, S. P., et Shlionsky, H. (1932). Arch. Neurol. Psychiat., Chicago, 27, 906.

Dercum, Fr. (1925). Ibid., 13, 651.

De Vos, L., et van Bogaert, L. (1934). Nederl. Tijdschr. Geneesk., 78, 4073.

Eicke, W. J. (1940). Arch. Psychiat. Nervenkr., 3, 514.

Feiling, A. (1923). Brain, 47, 421, 641.

Foix, Ch., et Nicolesco, J. (1925). "Les noyaux gris centraux et la région mésencéphalo sous optique," Masson, Paris.

Fünfgeld, E. (1929). Jhrb. Psychiat. Neurol., 40, 85.

Gamper, E. (1929). Ibid., 39, 39.

Glees, P. (1943). J. Neurol. Psychiat., 6, 92. (1945). Brain, 68, 331 .
Greenfield, J. G. (1934). Ibid., 57, 161.

Guillain, G., Mathieu, R., et Bertrand, J. (1926). Ann. de Méd., 20, 5.

Hallervorden, F. (1928). "Anatomie der Psychosen," J. Springer, Berlin, p. 1045.

Hallervorden, J., et Spatz, H. (1922). Z. ges. Neurol. Psychiat., 79, 254.

Hassler, R. (1937). Jhrb. Psychiat. Neurol., 48, 1, 387.

Hunt, J. R. (1917). Brain, 40, 58. (1933). Arch. Neurol. Psychiat., Chicago, 30, 1332.

Jakob, A. (1923). Die Extrapyramidal Erkrankungen, J. Springer, Berlin.

Kennard, M. A. (1944). J. Neurophysiol., 7, 127.

Ley, R. A. (1925). J. belge Neurol., 25, 92.

Lhermitte, J., et McAlpine, D. (1926). Brain, 49, 157.

Martin, J. P. (1945). Ibid., 68, 167.

Mettler, F. A., et Mettler, C. C. (1942). Ibid., 65, 242.

Meyers, R. (1942). Diseases of the Basal Ganglia. Research Publ., Ass. Research Nerv. Ment. Dis., 21, 602. Williams and Wilkins, Baltimore.

Norman, R. M. (1940). J. Neurol. Psychiat., 3, 311.

Papez, J. (1942). Diseases of the Basal Ganglia. Research Publ., Ass. Research Nerv. Ment. Dis., 21, 21. Williams and Wilkins, Baltimore.

Ranson, S.W. (1943). "Anatomy of the Nervous System," $7^{\circ}$ édit., W. B. Saunders, Philadelphia, p. 264. et Berry, C. (1941). Arch. Neurol. Psychiat., Chicago, 46, 504.

et Ranson, S. W., jun. (1941). Trans. Amer. neurol. Ass., 57, 168.

Scherer, H. J. (1933). Z. ges. Neurol. Psychiat., 145, 406.

Spatz, H. (1921). Münch. med. Wschr., 68, 1441. (1922). Z. ges. Neurol. Psychiat., 77, 261.

Spielmeyer, W. (1920). Ibid., 57, 312. (1926). Ibid., 101, 701.

van Bogaert, L. (1930). Rev. neurol., 54, 315. (1939-1940). Ibid., 72, 448.

- (1941). Mschr. Psychiat. Neurol., 103, 321. (1946). Ibid. (à paraître).

- et Scherer, H. J. (1936). Ibid., 93, 140.

Vincent, C., et van Bogaert, L. (1936). Rev. neurol., 65, 921.

Vogt, C. (1911). Jhrb. Psychiat. Neurol., 18, Suppt. 4, 479.

— et Vogt, O. (1920). Ibid., 25, Suppt. 3, 747.

$$
\text { (1937a). Ibid., 47, } 4 .
$$$$
\text { (1937b). Ibid., 48, } 1 .
$$

$$
\text { (1942). Ibid., 50, } 161 \text {. }
$$

Wilson, S. A. K. (1913). Brain, 36, 427.

Winkelman, N. W. (1932). Arch. Neurol. Psychiat., Chicago, 27, 1 . 